\title{
Regulation of GDSL Lipase Gene Expression by the MPK3/MPK6 Cascade and Its Downstream WRKY Transcription Factors in Arabidopsis Immunity
}

\author{
Xiaofei Han, ${ }^{1}$ Sen Li, ${ }^{1}$ Miao Zhang, ${ }^{1}$ Liuyi Yang, ${ }^{1}$ Yidong Liu, ${ }^{2}$ Juan $\mathrm{Xu},{ }^{1,+}$ and Shuqun Zhang ${ }^{2,+}$ \\ ${ }^{1}$ State Key Laboratory of Plant Physiology and Biochemistry, College of Life Sciences, Zhejiang University, Hangzhou, Zhejiang \\ 310058, China; and ${ }^{2}$ Division of Biochemistry, Interdisciplinary Plant Group, Bond Life Sciences Center, University of Missouri, \\ Columbia, MO 65211, U.S.A.
}

Accepted 5 December 2018.

\begin{abstract}
Mitogen-activated protein kinase (MAPK) cascades serve as unified signaling modules in plant development and defense response. Previous reports demonstrated an essential role of Arabidopsis GLIP1, a member of the GDSL-like-motif lipase family, in both local and systemic resistance. GLIP1 expression is highly induced by pathogen attack. However, the one or more signaling pathways involved are unknown. Here, we report that two pathogen-responsive MAPKs, MPK3 and MPK6, are implicated in regulating gene expression of GLIP1 as well as GLIP3 and GLIP4. After gain-of-function activation, MPK3 and MPK6 can strongly induce the expression of GLIP1, GLIP3, and GLIP4. Both GLIP1 and GLIP3 contribute to the plant resistance to Botrytis cinerea. WRKY33, a MPK3/MPK6 substrate, is essential for the MPK3/MPK6-dependent GLIP1 induction. In addition, WRKY2 and WRKY34, two close homologs of WRKY33, have a minor effect in MPK3/MPK6-regulated GLIP1 expression in B. cinerea-infected plants. Chromatin immunoprecipitation-quantitative polymerase chain reaction analysis demonstrated that the GLIP1 gene is a direct target of WRKY33. In addition, we demonstrated that MPK3/MPK6-induced GLIP1 expression is independent of ethylene and jasmonic acid, two important hormones in plant defense. Our results provide insights into the regulation of the GLIP family at the transcriptional level in plant immunity.
\end{abstract}

Plants have multifaceted defense systems that are either constitutive or inducible in response to pathogen invasion.

Sequence data from this paper are available in the Arabidopsis Genome Initiative or GenBank/EMBL databases under the following accession numbers: MPK3 (At3g45640), MPK6 (At2g43790), EF1 $\alpha$ (At5g60390), GLIP1 (At5g40990), GLIP2 (At1g539 40), GLIP3 (At1g53990), GLIP4 (At3g14225), GLIP5 (At1g53920), GLIP6 (At1g71120), GLIP7 (At5g15720), WRKY2 (At5g5 6270), WRKY33 (At2g38470), WRKY34 (At4g26440), ETRI (At1g66340), EIN2 (At5g03280), EIN3 (At3g20770), CTR1 (At5g03730), and COII (At2g39940), and ACT8 (At1g49240).

${ }^{\dagger}$ Corresponding authors: J. Xu; xujuan@zju.edu.cn and S. Zhang; Zhangsh@missouri.edu

Funding: This research was supported by grants from the Young Elite Scientist Sponsorship Program by CAST (2018QNRC001) and National Natural Science Foundation of China (31570297) to J. Xu and from the 111 Project (B14027) to S. Zhang.

*The $\boldsymbol{e}$-Xtra logo stands for "electronic extra" and indicates that eight supplementary figures and one supplementary table are published online.

() 2019 The American Phytopathological Society
Inducible defense responses are initiated after plant sensing of pathogen or microbe-associated molecular patterns or pathogen-originated effectors, which stimulates a set of early defense events, including activation of mitogen-activated protein kinases (MAPKs), flux of calcium ion, burst of reactive oxygen species, and changes in plant hormone biosynthesis, such as ethylene biosynthesis. These early defense responses play critical roles in signaling multiple intermediate and late events, including cell-wall modification, defense gene activation, hypersensitive-response cell death, phytoalexin accumulation, and finally lead to the induction of plant resistance (Bacete et al. 2018; Boller and Felix 2009; Coll et al. 2011; Dixon 2001; Glazebrook 2005; Jwa and Hwang 2017; Kotchoni and Gachomo 2006; Meng and Zhang 2013). Additionally, these localized defense responses can induce uninfected parts of plants to elicit systemic acquired resistance (SAR), conferring elevated resistance against subsequent pathogen challenge (Sticher et al. 1997). There are many up-to-date reviews available that GDSL-motif lipase/esterase 1 (GLIP1) possesses antimicrobial activity in local resistance and is also involved in SAR (Kim et al. 2013, 2014; Kwon et al. 2009; Oh et al. 2005).

GLIPs, a subfamily of lipolytic enzymes, consist of a characteristic GDSL motif that differs from the common lipases (Akoh et al. 2004). Like other esterase and lipase families, GLIPs possess the same invariant residues (serine, aspartic acid, and histidine) that make up the conserved catalytic triad. However, the classical lipases feature sequence $\mathrm{GxSxG}$ is replaced by GxSxxxxG, where the serine at the active sites is found to be relatively closer to the $\mathrm{N}$-terminus rather than near the center (Brick et al. 1995). Increasing evidence implicates that GLIPs play critical roles in plant immunity, especially in rice and Arabidopsis (Gao et al. 2017; Lee et al. 2009; Oh et al. 2005). There are seven GLIP members in the Arabidopsis genome (Oh et al. 2005). Secretome analysis identified Arabidopsis GLIP1 as a salicylic acid (SA)-responsive secreted protein (Oh et al. 2005). The protein sequences show that GLIP1 has 68 to $78 \%$ identity to GLIP2, GLIP3, and GLIP4, while it is 32 to $52 \%$ identical to GLIP5, GLIP6, and GLIP7. It has been shown that GLIP2 negatively regulate auxin signaling to combat invading pathogens (Lee et al. 2009). At present, the functions of the other five GLIPs are poorly understood. GLIP1 is highly induced after pathogen invasion. Nonetheless, the one or more signaling pathways involved are lacking.

MAPK cascades, conserved signaling modules during plant immunity, convert external stress signal from upstream receptors and sensors into a variety of cellular responses (Bigeard and Hirt 2018; Chen et al. 2001; Colcombet and Hirt 2008; 
Dóczi et al. 2012; Pitzschke 2015; Taj et al. 2010; Xu and Zhang 2015a; Zhang et al. 2018). Each typical MAPK cascade generally contains three tiers of protein kinases, including a MAPK kinase kinase (known as MAPKKK or MEKK), its downstream MAPK kinase (known as MAPKK or MEK), and the terminal MAPK (Rodriguez et al. 2010). In Arabidopsis thaliana, there are four MAPKs that have been connected to immunity, including MPK3, MPK4, MPK6, and MPK11 (Bethke et al. 2012; Meng and Zhang 2013). Among them, MPK3 and MPK6, which are mostly functionally redundant, act downstream of two redundant MAPKKs, MKK4 and MKK5 (Asai et al. 2002; Meng and Zhang 2013; Pedley and Martin 2005; Wang et al. 2007). Another separate pathogenresponsive MAPK cascade consists of MPK4, two redundant MAPKKs, MKK1 and MKK2, along with the top tier MEKK1 (Gao et al. 2008; Ichimura et al. 2006; Kong et al. 2012; Meng and Zhang 2013; Qiu et al. 2008; Suarez-Rodriguez et al. 2007). At present, the kinases upstream of MPK11 are unclear.

Our recent studies demonstrate that MPK3 and MPK6 positively regulate defense responses, such as the induction ethylene biosynthesis (Guan et al. 2015; Han et al. 2010; Joo et al. 2008; Li et al. 2012; Liu and Zhang 2004), defense gene activation (Meng et al. 2013), camalexin production (Mao et al. 2011; Ren et al. 2008), indole glucosinolate (IGS) biosynthesis (Xu et al. 2016), stomatal immunity ( $\mathrm{Su}$ et al. 2017), and photosynthesis inhibition (Su et al. 2018). The multifunctionality of MPK3 and MPK6 in plant disease resistance can be attributed to the multiple MAPK substrates. For instance, MPK3 and MPK6 are able to phosphorylate several WRKY transcription factors, including WRKY2, WRKY33, and WRKY34 (Guan et al. 2014; Mao et al. 2011; Ueda et al. 2017). WRKY33 was shown not only to positively regulate camalexin biosynthetic gene expression but it also promotes the expression of ACS2 as well as ACS6 downstream of MPK3 and MPK6 in plant immunity (Li et al. 2012; Mao et al. 2011). As the substrate of MPK3 and MPK6, both WRKY2 and WRKY34 transcription factors regulate pollen development and WRKY2 is also capable of upregulating $W O X-8$ expression to polarize the zygote (Guan et al. 2014; Ueda et al. 2017).

It is demonstrated in this report that pathogen-induced expression of several members of the GLIP family, including GLIP1, GLIP3, and GLIP4, depends on the MPK3/MPK6 signaling pathway. In the conditional gain-of-function $G V G$ NtMEK $2^{D D}(D D)$ and Botrytis cinerea-inoculated Arabidopsis, GLIP1 expression is highly induced after MPK3 and MPK6 activation. We further found that both GLIP1 and GLIP3 play important roles in Arabidopsis resistance to $B$. cinerea. WRKY33, a MPK3/MPK6 substrate, is responsible for turning on the MPK3/MPK6-dependent GLIP1 expression. Additionally, WRKY2 and WRKY34, two close homologs of WRKY33, are also involved in GLIP1 induction in B. cinerea-infected plants. Chromatin immunoprecipitation-quantitative polymerase chain reaction (ChIP-qPCR) analysis demonstrated GLIP1 gene is a direct target of WRKY33. Furthermore, it is found that the expression of GLIPl is independent of ethylene and jasmonic acid (JA) signaling. Our results provide insights into the regulation and the importance of the GLIP family in plant immunity.

\section{RESULTS}

\section{Induction of GLIP expression} after MPK3/MPK6 activation in the conditional gain-of-function $D D$ Arabidopsis plants.

Expression profiling of the conditional gain-of-function $D D$ plants (Ren et al. 2002; Yang et al. 2001) revealed that GLIP1 and its homologs GLIP3 and GLIP4 are extremely induced upon dexamethasone (DEX) treatment (Tsuda et al. 2013). Previous studies demonstrated that GLIP1 is a pathogen- and SA-responsive secreted protein and is involved in Arabidopsis resistance to biotrophic and necrotrophic pathogens (Kim et al. 2013, 2014; Kwon et al. 2009; Oh et al. 2005). However, the signaling pathways underlying the induction of GLIP1 gene expression remain unclear. As a result, we decided to further investigate the regulation of GLIPs by the MPK3/MPK6 signaling pathway. As the first step, we confirmed the induction of these GLIPs after MPK3/MPK6 activation, using quantitative reverse transcription (RT)-PCR. The levels of GLIP1, GLIP3, and GLIP4 expression were greatly induced in $D D$ plants in response to DEX treatment (Fig. 1). In contrast, GLIP2, GLIP5, GLIP6, and GLIP7 expression could be reliably detected but at very low levels and not inducible. We calculated the expression levels of GLIP genes versus that of EFl $\alpha$ and were able to compare relative expression levels between distinct GLIP genes through this calculation (Fig. 1A). To better assess gene activation levels, we also calculated their induction fold over their basal levels. As shown in Figure 1B, transcripts of GLIP1, GLIP3, and GLIP4 were induced by approximately 4,500-, 300-, and 100-fold, respectively. Consistently, no increases of GLIP2, GLIP5, GLIP6, and GLIP7 expression were observed. These results demonstrate that the transcription of GLIP1, GLIP3, and GLIP4 genes can be activated after activation of MPK3/MPK6 in DD Arabidopsis.

\section{Induction of GLIP1, GLIP3, and GLIP4 gene expression is dependent on the MPK3/MPK6 pathway.}

To determine whether GLIP1, GLIP3, and GLIP4 gene induction depends on MPK3 or MPK6 in $D D$ plants, we analyzed the expression of GLIP1, GLIP3, and GLIP4 in DD, DD mpk3, and $D D$ mpk6 plants. We found that absence of either $M P K 3$ or MPK6 compromised the induction of GLIP1, GLIP3, and GLIP4 expression in $D D$ plants after DEX application (Fig. 2). Moreover, GLIP4 expression in DD mpk6 plants was higher at $24 \mathrm{~h}$ after DEX treatment than that in $D D$ plants, possibly since delayed cell death occurred in the $D D$ mpk6 plants. These data support the idea that endogenous MPK3/MPK6 activation is responsible for GLIP1, GLIP3, and GLIP4 gene induction in DD seedlings.

Botrytis cinerea, a necrotrophic pathogen, highly activates MPK3 and MPK6 in Arabidopsis (Han et al. 2010). It was previously reported that the expression of Arabidopsis GLIPI is induced by Alternaria brassicicola (Kwon et al. 2009; Oh et al. 2005), a necrotrophic pathogen that has the same lifestyle as B. cinerea (Glazebrook 2005). As a result, we examined whether $B$. cinerea can induce GLIP1, GLIP3, and GLIP4 gene expression and whether this induction is MPK3/MPK6dependent. As indicated in Figure $3 \mathrm{~A}$ to $\mathrm{C}$, the GLIP1, GLIP3, and GLIP4 expression was obviously induced in Arabidopsis that was inoculated with $B$. cinerea spores. To obtain loss-of-function data to further understand the function of MPK3/MPK6 cascade in B. cinerea-induced GLIP gene activation, we first examined its expression in wild type (Col-0) and $m p k 3$ and $m p k 6$ single mutants. Loss of function of $M P K 3$ was associated with a mild decrease of GLIP1 induction (Fig. $3 \mathrm{~A}$ ), whereas no significant reduction was detected in $m p k 6$ mutant. The subtle change in mpk3 and mpk6 single mutants could result from functional redundancy.

MPK3 and MPK6 have mostly redundant impacts on a variety of biological processes, for instance, embryogenesis (Wang et al. 2007; Zhang et al. 2017). Mutation of both MPK3 and $M P K 6$ results in an embryo-lethal phenotype. We utilized a chemical genetic method to circumvent this problem and generate a conditional double mutant system for functional analyses (Bishop et al. 2000). A chemical-sensitized MPK6 variant, 
MPK6 ${ }^{\mathrm{YG}}$, was used to successfully rescue the $m p k 3$ mpk6 lethal phenotype, and the generating plants were designed as MPK6SR plants (genotype: mpk3 mpk6 $\left.P_{M P K \sigma}: M P K 6^{Y G}\right)(\mathrm{Xu}$ et al. 2014). In the absence of 4-amino-1-tert-butyl-3-(1'naphthyl)pyrazolo [3,4-d]pyrimidine (NA-PP1) inhibitor, MPK6SR plants grow and develop normally, similar to $m p k 3$ single mutant. When we apply NA-PP1 to MPK6SR plants, MPK6 ${ }^{\mathrm{YG}}$ activity is inhibited in vivo, resulting a $m p k 3 \mathrm{mpk6}$ double-activity null mutant. NA-PP1 is an analog of PP1 kinase inhibitor, which has a bulkier side chain. As a result, it cannot access the ATP binding site of a wild-type kinase (Bishop et al. 2000), ensuring the specificity of this approach. Likewise, we bred a rescued $m p k 3$ mpk6 double mutant employing a NAPP1-sensitive MPK3 variant, MPK $3{ }^{\mathrm{TA}}$ (genotype: $m p k 3$ mpk6 $P_{M P K 3}: M P K 3^{T A}$, designed as MPK3SR plants) (Xu et al. 2016).

In MPK6SR plants without NA-PP1 treatment (equivalent to the $m p k 3$ single mutant), induction of GLIP1 expression was partially compromised (Fig. 3D), consistent with what we observed in the mpk3 single mutant (Fig. 3A). In contrast, in the presence of NA-PP1, GLIP1 induction in MPK6SR plants was severely reduced after $B$. cinerea inoculation (Fig. 3D). In $M P K 3 S R$ plants without NA-PP1, induction of GLIP1 expression was not affected, similar to the mpk6 single mutant, whereas $B$. cinerea-induced GLIP1 gene activation was dramatically reduced in $M P K 3 S R$ plants after NA-PP1 treatment. These data demonstrate the redundant function of MPK3 and MPK6 in induction of GLIP1 expression after B. cinerea inoculation. At a late timepoint such as $24 \mathrm{~h}$ after pathogen inoculation, GLIP1 induction in NA-PP1 pretreated MPK3SR and MPK6SR increased, possibly a result of loss of NA-PP1 inhibitor due to plant metabolism. Alternatively, this residual activation could be a result of one or more MPK3/MPK6independent pathways. We also examined GLIP3 and GLIP4 gene expression in MPK6SR and MPK3SR plants inoculated with $B$. cinerea. In Figure $3 \mathrm{E}$ and $\mathrm{F}$, the induction levels of GLIP3 and GLIP4 expression were severely compromised in the loss-of-function plants, similar to the expression pattern of GLIP1. These results further support the role of MPK3 and MPK6 in the expression regulation of GLIP1, GLIP3, and GLIP4 in plant immunity.

\section{GLIP1 and GLIP3 contribute}

to the plant resistance to $B$. cinerea.

The MPK3/MPK6-dependent high-level induction of GLIP1, GLIP3, and GLIP4 genes prompted us to test the resistance of glip1, glip3, and glip4 mutant plants against $B$. cinerea. We obtained a T-DNA insertion line for GLIP3 (SALK_201623C, named as glip3-1) and two T-DNA insertion lines for GLIP4 (WiscDsLox489-492B15 and SALK_048818, named glip4-1 and glip4-2, respectively) (Supplementary Fig. S1). These mutant plants did not express GLIP3 or GLIP4 transcripts, respectively, as determined by RT-PCR analysis. The glipl-1 and glip1-2 mutants were the same as previously reported (Oh et al.

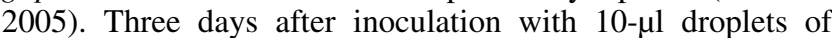
$B$. cinerea spore suspension, the disease phenotype in Col-0 and mutant plants was observed and the lesion sizes were measured. B. cinerea caused much bigger lesions in the glip1-1 and glip12 mutant plants than in Col-0 control plants (Fig. 4A and B). The glip3-1 mutant plants also exhibited larger lesions compared with Col-0 but smaller than those on glip1-1 and glipl-2 mutants. In contrast, mutation of GLIP4 did not alter the $B$. cinerea susceptibility. These results demonstrate the importance of GLIP1 and GLIP3 in plant resistance to $B$. cinerea.

\section{WRKY33 is involved}

in the MPK3/MPK6-regulated GLIP1 gene activation.

Because of the importance of GLIP1 in plant immunity (Kim et al. 2013, 2014; Kwon et al. 2009; Oh et al. 2005) and its

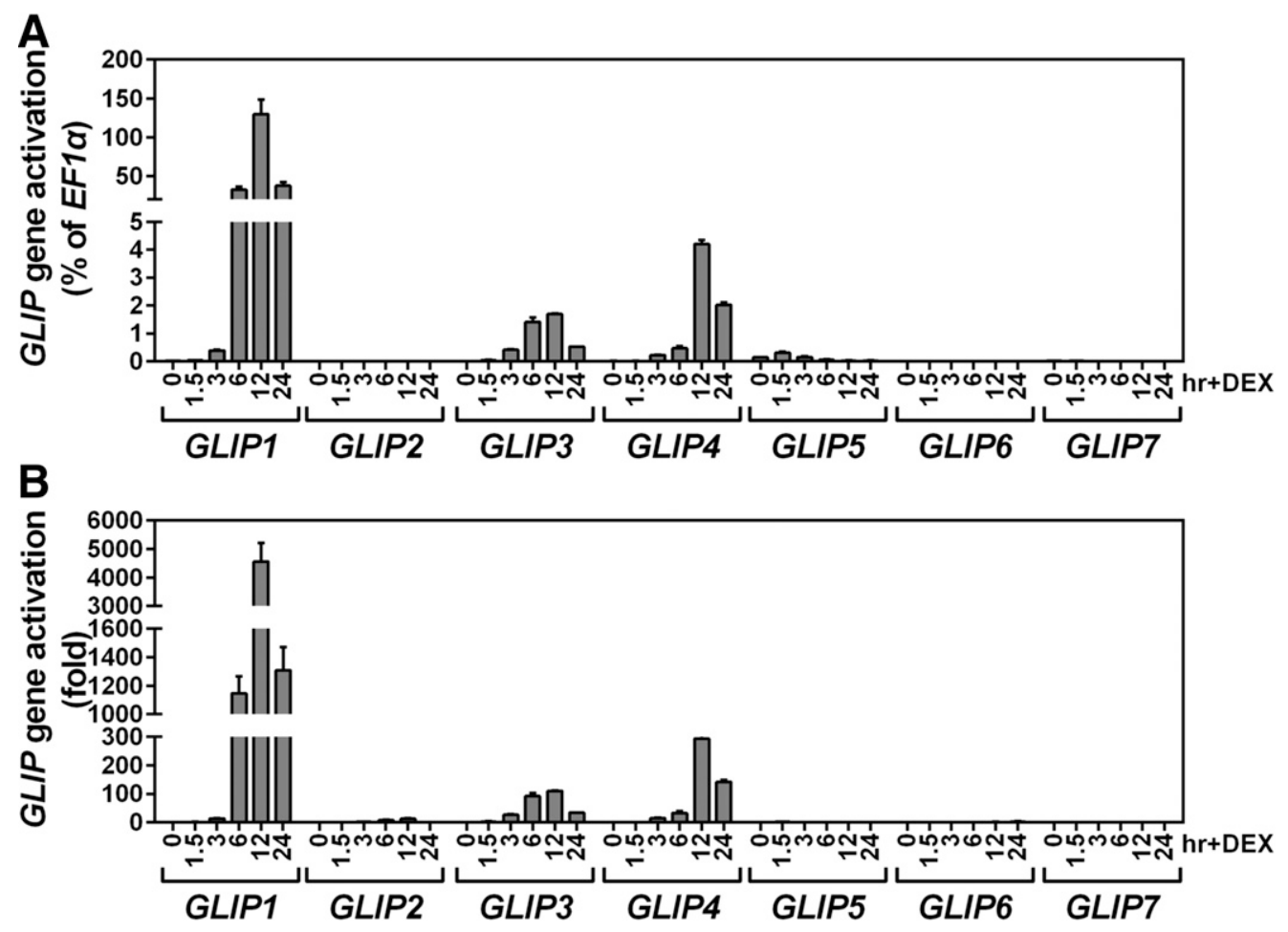

Fig. 1. Activation of GDSL LIPASE (GLIP) gene expression after MPK3/MPK6 activation in the gain-of-function GVG-NtMEK2 ${ }^{D D}$ (DD) Arabidopsis seedlings. Conditional gain-of-function DD Arabidopsis seedlings (12 days old) were treated with $1 \mu \mathrm{M}$ dexamethasone (DEX). Samples were collected at indicated times. Real-time polymerase chain reaction was used to quantify the expressions of all seven GLIP genes. A, GLIP gene activation was expressed as the percentage of $E F 1 \alpha$ expression, allowing the comparison of expression levels among different GLIP genes. B, Induction of GLIP gene expression (fold of induction relative to the level before treatment) was calculated by the $2^{-\Delta \Delta \mathrm{Ct}}$ method. In both calculations, the expression of $E F 1 \alpha$ was used as a reference. Error bars represent standard deviations $(n=3)$. 
higher induction levels (Figs. 1, 2, and 3), we focused on this isoform for more detailed analysis. WRKY33, a MPK3/MPK6 substrate, functions as the transcriptional activator in promotion of camalexin biosynthetic genes expression and camalexin generation in Arabidopsis (Mao et al. 2011). To determine whether WRKY33 also participates in the modulation of GLIP1 gene expression downstream of the MPK3/MPK6 cascade, we performed real-time qPCR to quantify its expression in $D D$ and DD wrky33-1 plants after DEX treatment. As seen in Figure 5A, the activation of GLIP1 gene expression by $D D$ transgene was greatly reduced in the background of wrky33-1 mutant. Examination of $D D$ wrky33-2 plants gave a similar result (Supplementary Fig. S2A). Based on the residual level of GLIP1 gene activation in $D D$ wrky33 plants, we can also conclude that, in addition to WRKY33, one or more other transcription factors are also involved and can partially replace WRKY33 in its absence.

We then tested whether pathogen-induced GLIP1 expression requires WRKY33 by quantifying the expression of GLIP1 in B. cinerea-induced Col-0 and wrky33-1 plants. As expected,

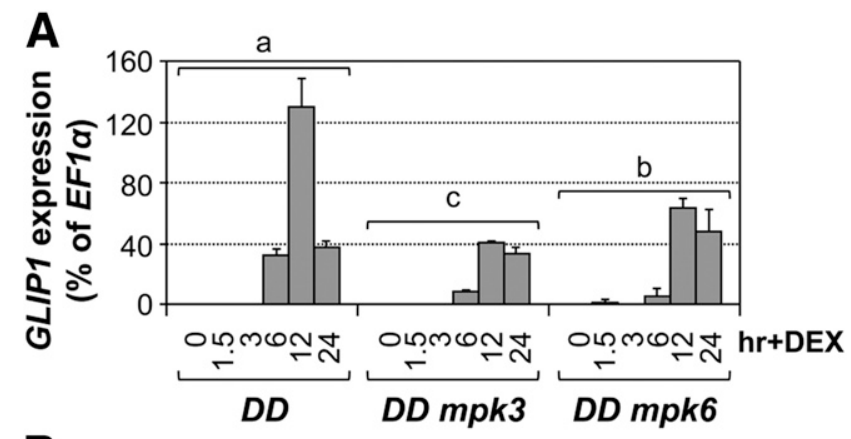

B
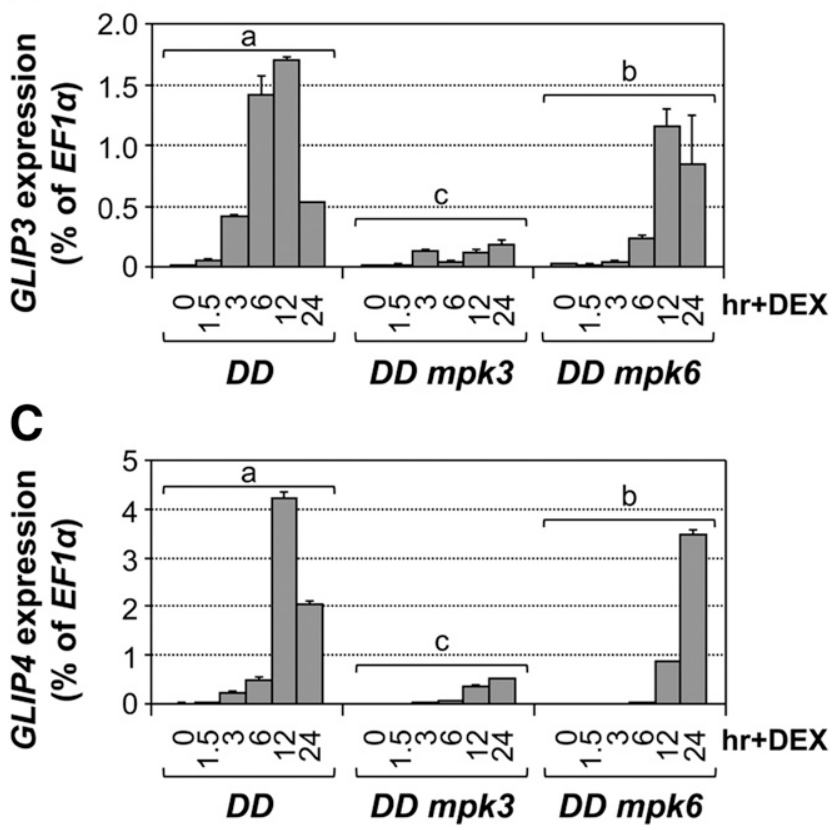

Fig. 2. Activation of GLIP1, GLIP3, and GLIP4 gene expression in gain-offunction $G V G-N t M E K 2^{D D}(D D)$ seedlings is dependent on MPK3 and MPK6. The 12-day-old $D D, D D m p k 3$, and $D D$ mpk6 seedlings were treated with $1 \mu \mathrm{M}$ dexamethasone (DEX). Samples were collected at indicated times. A, Real-time polymerase chain reaction was used to quantify the expressions of GLIP1, B, GLIP3, and C, GLIP4 genes. The GLIP genes transcript levels were calculated as a percentage of the EF1 $\alpha$ transcript. Error bars represent standard deviations $(n=3)$. Two-way analysis of variance was performed to compare the levels of GLIP gene expressions in different genotypes after DEX treatment. Different lowercase letters above the brackets represent statistically significant differences $(P<0.05)$. the induction of GLIP1 mRNA by $B$. cinerea infection was reduced in the wrky33-1 mutant in comparison with the Col0 control (Fig. 5B). Examination of wrky33-2 mutant gave a similar result. The remaining GLIP1 induction detected in the B. cinerea-induced wrky33 mutant once more points to the involvement of one or more other transcription factors.

\section{Botrytis cinerea-induced GLIP1 expression is partially dependent on WRKY2 and WRKY34.}

Our previous research demonstrated that WRKY34 and WRKY2, two highly homologous transcription factors with WRKY33, function in pollen development downstream of MPK3/MPK6 (Guan et al. 2014). MPK3/MPK6-dependent phosphorylation of WRKY34 is crucial to its role in the development of plant pollen. Both WRKY34 and WRKY2 have broad expression patterns, despite the fact that $W R K Y 34$ expression is much higher in pollen (Guan et al. 2014), suggesting their one or more roles in other cells and tissues. To determine whether WRKY2 and WRKY34 are responsible for the residual GLIP1 induction in wrky33 mutant, we crossed wrky2 wrky34 double mutants into wrky33 background and identified a wrky 2 wrky33 wrky34 triple mutant. We then monitored the expression of GLIP1 in Col-0, wrky33 single-, wrky2 wrky34 double-, and wrky 2 wrky33 wrky34 triple-mutant plants at different times after infection by $B$. cinerea. There was a slight reduction of B. cinerea-triggered GLIP1 induction in wrky 2 wrky 34 doublemutant plants in comparison with Col-0 (Fig. 6). We also found that GLIP1 expression level in wrky2 wrky33 wrky34 triple mutant was lower than that in the wrky33 single mutant and the wrky2 wrky34 double mutant after $B$. cinerea infection, confirming the involvement of WRKY2 and WRKY34 in B. cinereainduced GLIP1 expression. This result can explain the residual induction of GLIP1 in wrky33 mutant plants inoculated with B. cinerea (Fig. 5B). We also examined the resistance phenotypes of Col-0, wrky33, wrky2 wrky34, and wrky2 wrky33 wrky34 mutant plants in response to $B$. cinerea (Supplementary Fig. S3). In comparison with Col-0 control plants, wrky33 single mutant plants developed more extensive necrosis. In contrast, wrky 2 wrky34 double mutant plants showed wild type-like resistance to B. cinerea. Although wrky2 wrky33 wrky34 triple mutant plants were more susceptible to $B$. cinerea than Col-0 plants, no obvious difference between the triple mutants and wrky33 single mutants was observed. These results indicated that WRKY33 play a more important role in resistance to $B$. cinerea and the regulation of GLIP1, whereas its two close homologs, WRKY2 and WYKY34, play a minor role in regulating GLIP1 expression in response to $B$. cinerea infection.

\section{WRKY33 binds to the W-boxes}

in the promoter of GLIP1 gene in vivo.

Next, we further investigated the detail function of WRKY33 in regulating the expression of the GLIPl gene. Sequence analysis identified three $\mathrm{W}$-box elements in the $2.2-\mathrm{kb}$ promoter region of the GLIP1 gene (Fig. 7B). We then performed ChIPqPCR to examine whether GLIPl is a direct target of the WRKY33 transcription factor in plant defense. WRKY33 protein has five MAPK phosphorylation sites in the $\mathrm{N}$ terminus, including serine-54, serine-59, serine-65, serine-72, and serine-85 (Mao et al. 2011). For this experiment, we generated transgenic plants expressing WRKY $33^{\mathrm{SD}}$, a phosphomimicking form of WRKY33 with all five serine (S) residues mutated to aspartic acid (D) (Fig. 7A), in wrky33 mutant background. The WRKY33 $3^{S D}$ transgene was driven by the $35 \mathrm{~S}$ promoter and was attached with a 4 myc epitope tag at the $\mathrm{N}$ termini. As indicated in Figure 7C, in 35S:4myc$W R K Y 33^{S D} /$ wrky33 plants, the antimyc antibody was able to highly enrich the W-box-containing GLIPl promoter region 
via coimmunoprecipitation with the 4 myc-WRKY $33^{\mathrm{SD}}$ protein. In contrast, in control reactions, immunoglobulin $\mathrm{G}(\mathrm{IgG})$ antibody failed to enrich the GLIPI gene promoter. As another control, both antimyc and IgG antibody failed to coimmunoprecipitate GLIPI gene promoter sequence from the nontransgenic Col-0 plants. We also performed ChIP-qPCR in $35 S$ : $4 m y c-W R K Y 33^{W T} /$ wrky33 plants. As shown in Supplementary Figure S4, the wild-type form of WRKY33 exhibits similar binding activity to the GLIP1 promoter as does WRKY33 ${ }^{\mathrm{SD}}$, which is consistent with our previous conclusion that phosphorylation of WRKY33 by MPK3/MPK6 does not alter its DNA-binding ability to the W-box cis-element (Mao et al.
2011). These in-vivo data indicated the direct binding of WRKY33 to the GLIP1 promoter.

\section{Ethylene- and JA-independent regulation}

of GLIP1 expression in the MPK3/MPK6 cascade.

It was reported that pathogen-induced GLIP1 expression involves ethylene pathways (Kim et al. 2014; Oh et al. 2005). Plants that are inoculated with $B$. cinerea spores or $D D$ plants that are treated with DEX exhibit enhanced levels of ethylene ( $\mathrm{Li}$ et al. 2012; Liu and Zhang 2004). To detect whether the induction of GLIP1 gene expression observed in Figures 1, 2A, 3A, and 3D relies on ethylene sensing or signaling, we tested GLIP1
A

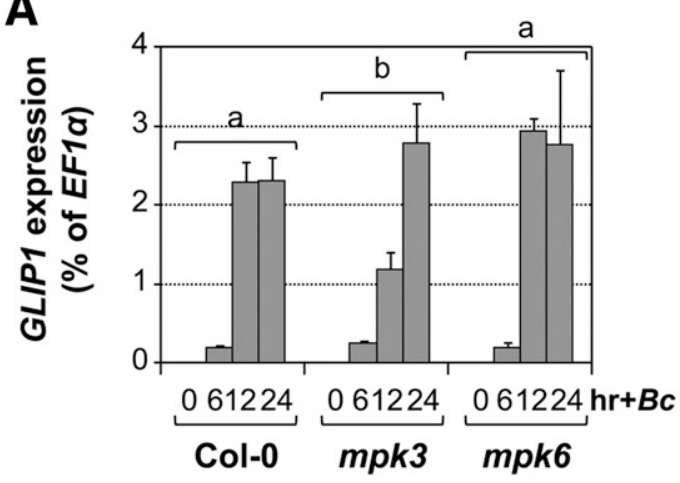

D

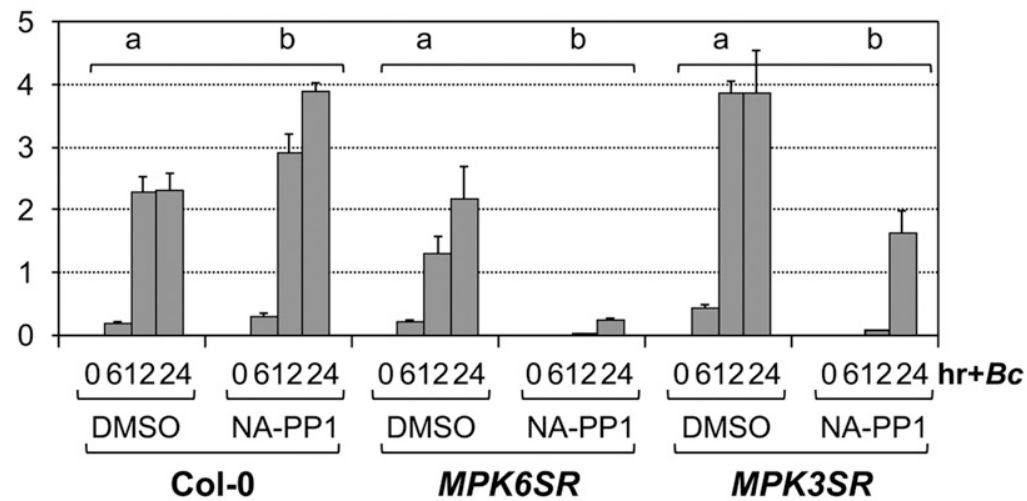

B

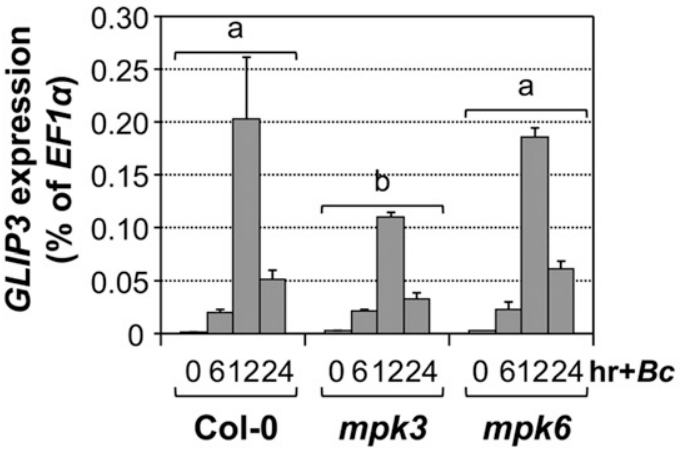

E

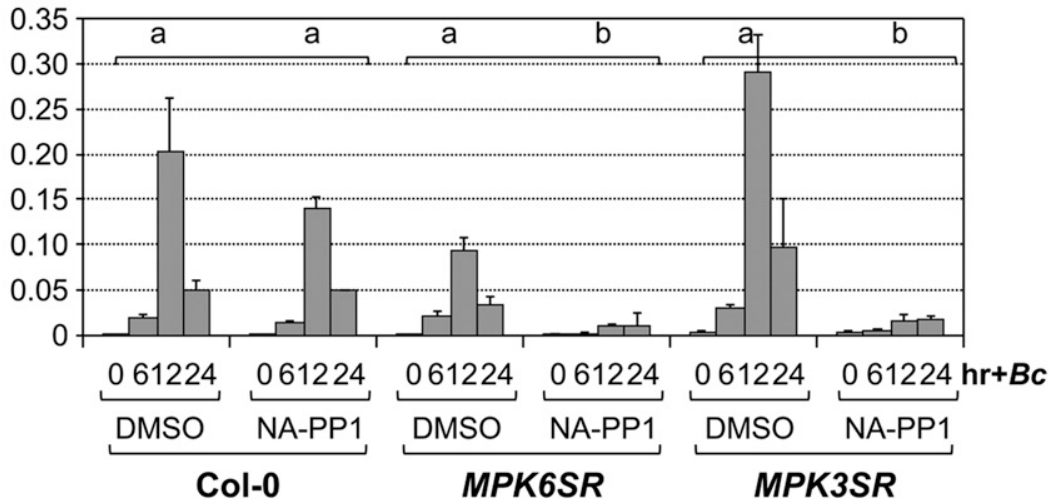

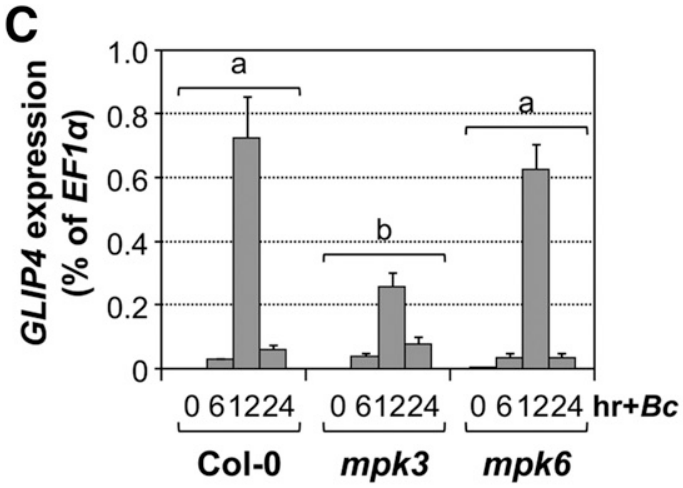

$\mathbf{F}$

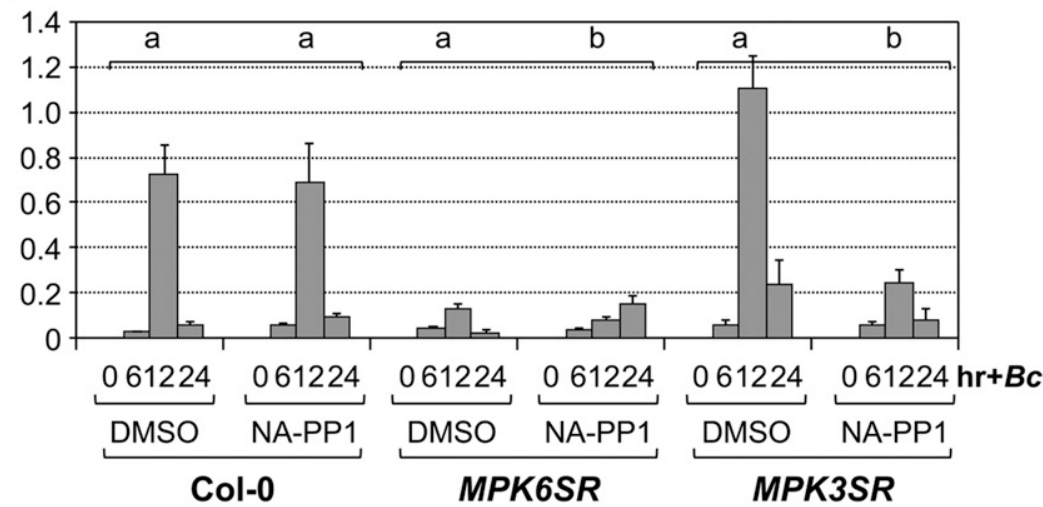

Fig. 3. Activation of Botrytis cinerea-induced GLIP1, GLIP3, and GLIP4 genes is dependent on functional MPK3 and MPK6. A to C, Wild-type (Col-0), $m p k 3$, and $m p k 6$ seedlings (12 days old) were inoculated with B. cinerea (Bc) spores, and $\mathbf{D}$ to $\mathbf{F}, 12$-day-old wild-type (Col-0) and chemical-genetically rescued $m p k 3$ mpk6 double mutant (MPK6SR genotype: $m p k 3$ mpk6 $P_{M P K 6}: M P K 6^{Y G}$; MPK3SR genotype: $m p k 3$ mpk6 $P_{M P K 3}: M P K 3^{T A}$ ) seedlings were inoculated with $B$. cinerea spores after pretreatment with 4-amino-1-tert-butyl-3-(1'-naphthyl)pyrazolo [3,4-d]pyrimidine (NA-PP1) (5 $\mu \mathrm{M}$ final concentration) or dimethyl sulfoxide (DMSO), the solvent of NA-PP1 stock solution, for $30 \mathrm{~min}$. Samples were collected at indicated times. Real-time polymerase chain reaction was used to quantify the expressions of GLIP1 (A and D), GLIP3 (B and E), and GLIP4 (C and F) genes. GLIP gene transcript levels were calculated as a percentage of the EF1 $\alpha$ transcript. Error bars represent standard deviations $(n=3)$. Two-way analysis of variance was performed to compare the expression levels of GLIP genes in different genotypes after $B$. cinerea inoculation. Different lowercase letters above the brackets represent statistically significant differences $(P<0.05)$. 
expression in $D D, D D$ etrl-1, DD ein2, and $D D$ ein3 plants treated with DEX. Although we detected a small decrease in GLIP1 expression in DD ein2 plants at $12 \mathrm{~h}$ after DEX treatment, GLIP 1 expression was not reduced at other timepoints. Furthermore, there was no decrease in GLIP1 induction in DD etr $1-1$ and DD ein3 plants (Fig. 8A), suggesting that activation of the MPK3/MPK6 pathway activates the expression of the GLIPI gene independent of ethylene sensing or signaling.

We further investigated the expression of the GLIPl gene in Col-0, etr1-1, ein2, ein3, and ctr1 plants after B. cinerea challenge. No decrease in GLIP1 activation was observed in etr1-1, ein2, or ein3 mutant after infection by B. cinerea (Fig. $8 \mathrm{~B})$. Instead, GLIP1 induction in these mutants was significantly higher. All of these indicated that pathogen-induced expression of GLIPI is not ethylene-dependent but ethylenesensing or -signaling is required to limit the levels of GLIP1 gene activation. Alternatively, the lack of ethylene sensing or signaling blocks a subset of defense responses, which allows deployment of more resources in activation of ethylene-independent defense responses such as GLIPI activation. We also compared the basal levels of GLIP1 transcript in Col-0, etr1-1, ein2, ein3, and ctr1 plants (Supplementary Fig. S5). Although the differences were significant, the fold changes are minimal, around twofold. In the constitutive ethylene-response mutant $c t r l$, the minimal increase in GLIP1 transcript level indicates that constitutive activation of ethylene signaling in $c t r l$ is not sufficient to activate GLIP1 expression. Consistent with this, 1 and $10 \mu \mathrm{M}$ 1-aminocyclopropane-1-carboxylic acid (ACC) treatment resulted in only two- and 3.5-fold induction of GLIP1 expression in
Col-0, respectively (Supplementary Fig. S6), suggesting a subtle role of ethylene in GLIP1 expression induction. Taken together, these findings show that GLIP1 expression induced by MPK3/MPK6 is independent of ethylene.

MPK6 was reported to be an essential regulator in Arabidopsis JA signaling pathway (Takahashi et al. 2007), although earlier research in our lab indicated that the activation of SIPK and WIPK, tobacco homologs of Arabidopsis MPK6 and MPK3, respectively, do not elevate JA level in tobacco (Kim et al. 2003). To investigate whether JA signaling pathway functions in the enhanced expression of GLIP1 after MPK3/MPK6 activation, we examined GLIP1 expression in $D D$ and $D D$ coil plants. COII, an F-box protein, is a coreceptor of JA and essential to JA-regulated responses (Gfeller et al. 2010). As indicated in Figure 8C, the GLIP1 expression was not significantly affected in $D D$ coil plants at 0,6 , and $12 \mathrm{~h}$, although its level was slightly reduced at $24 \mathrm{~h}$ in $D D$ coil in comparison with $D D$. We also analyzed the expression of GLIP1 in Col-0 and coil mutant plants after $B$. cinerea inoculation. Loss of COI1 was not sufficient to block GLIP1 expression (Fig. 8D). To the contrary, GLIP1 expression was higher in coil at $24 \mathrm{~h}$ after $B$. cinerea invasion. Furthermore, the level of GLIP1 transcript was not induced in Col-0 after methyl jasmonate (MeJA) treatment. Thus, we can conclude that JA signaling is not related to the MPK3- and MPK6-dependent induction of GLIP1.

\section{DISCUSSION}

The function of GLIP1 in Arabidopsis immunity has been characterized recently (Kim et al. 2013, 2014; Kwon et al.
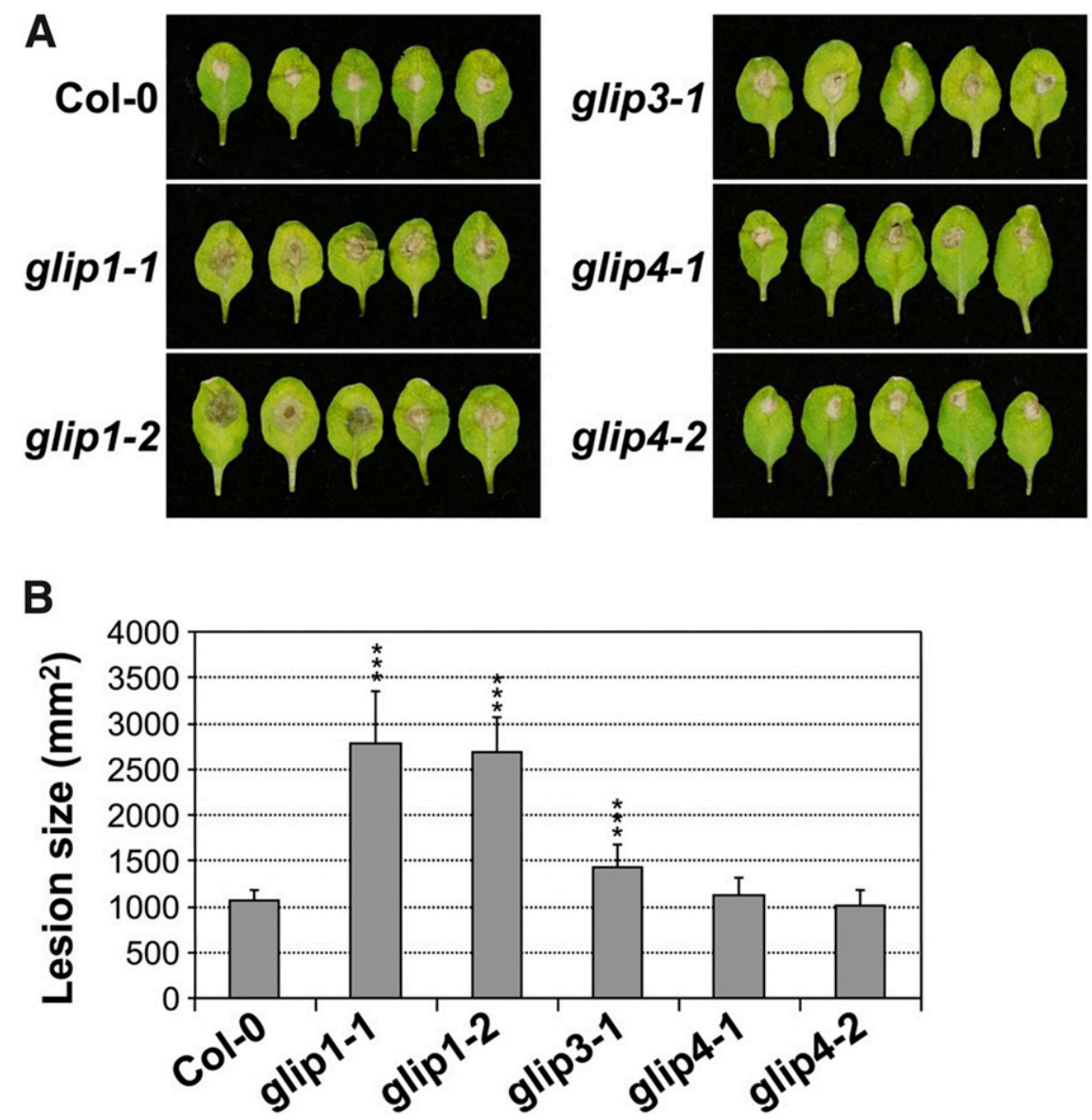

Fig. 4. Resistance phenotype in glip1, glip3, and glip4 mutant plants inoculated with Botrytis cinerea. The 4-week-old wild-type (Col-0), glip1-1, glip1-2 glip3-1, glip4-1, and gip4-2 plants grown under short-day conditions were drop-inoculated with $10 \mu 1$ of $B$. cinerea spore suspension $\left(5 \times 10^{5}\right.$ spores/ml). Disease symptoms were scored 3 days after inoculation. A, Necrotic lesion phenotypes. B, Measurement of lesion size. Error bars represent standard deviations $(n=10)$. Asterisks represent significant differences from Col-0 plants (Student's $t$ test, asterisks $[* * *]$ indicate $P<0.001)$. 
2009; Oh et al. 2005). High induction of GLIP1 expression is found during the plant immune process. However, the molecular mechanism underlying the induction of GLIP1 gene expression is unclear. In this report, the positive regulation of GLIP1 expression by MPK3 and MPK6, two important pathogen-responsive MAPKs, is investigated (Figs. 1, 2, and 3). It is also revealed that the implication of WRKY33, a known MPK3/MPK6 substrate (Mao et al. 2011), in B. cinerea triggered GLIPl induction (Fig. 5). ChIP-qPCR analysis demonstrated that the GLIP1 gene is a direct target of WRKY33 (Fig. 7). In addition, WRKY2 and WRKY34, two WRKY33 homologs downstream of MPK3 and MPK6 during pollen development (Guan et al. 2014), are involved in the residual activation of GLIP1 expression in the wrky33 mutant (Fig. 6), suggesting that these two WRKYs can either substitute partially the role of WRKY33 in its loss or function redundantly with WRKY33, as illustrated in our working model (Supplementary Fig. S7).

\section{Redundant function of MPK3 and MPK6 \\ in B. cinerea-induced GLIP expression.}

Gain-of-function DD-induced GLIP1, GLIP3, and GLIP4 gene expression was compromised by a single mutation of $M P K 3$ or $M P K 6$, whereas mutation of both $M P K 3$ and $M P K 6$ is required to compromise $B$. cinerea-triggered GLIP1, GLIP3, and GLIP4 gene activation (Figs. 2 and 3). We previously observed a similar phenomenon in MPK3/MPK6 cascademediated ACS2 and ACS6 gene activation and camalexin
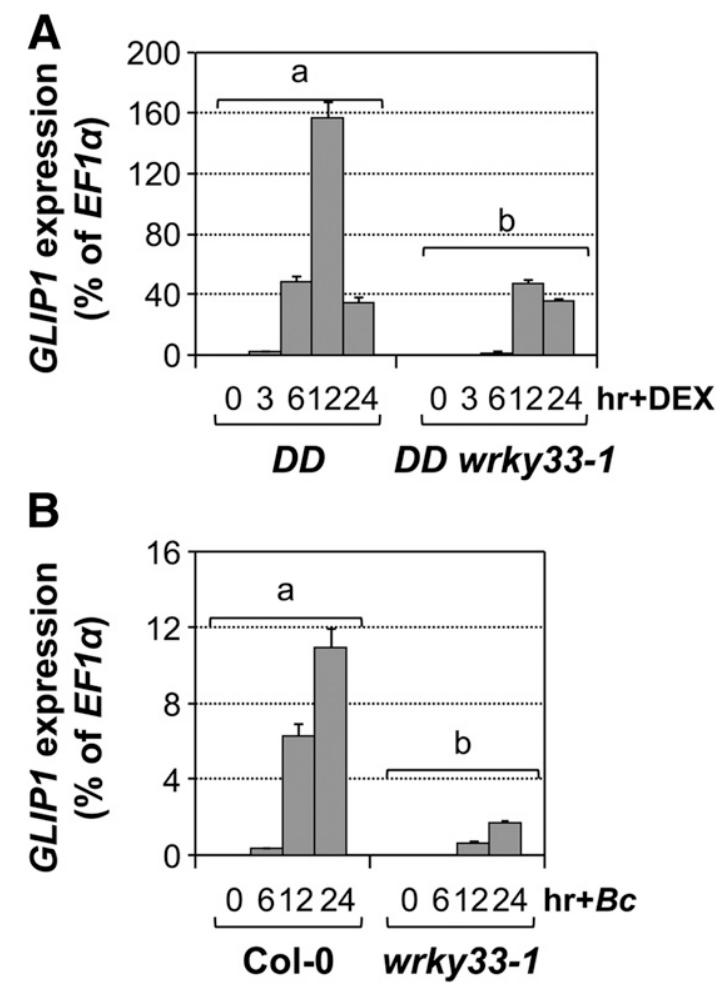

Fig. 5. WRKY33 is involved in the MPK3/MPK6-regulated GLIP1 gene activation. A, GVG-NtMEK2 ${ }^{D D}(D D)$ and $D D$ wrky33-1 seedlings (12 days old) were treated with $1 \mu \mathrm{M}$ dexamethasone (DEX), and B, 12-day-old wild-type (Col-0) and wrky33-1 seedlings were treated with Botrytis cinerea $(B c)$ spores. Samples were collected at indicated times. Real-time polymerase chain reaction was used to quantify the expression of GLIPI gene. GLIP1 transcript level was calculated as a percentage of the EFI $\alpha$ transcript. Error bars represent standard deviations $(n=3)$. Two-way analysis of variance was performed to compare the level of GLIP1 gene expression in different genotypes after DEX or $B$. cinerea treatment. Different lowercase letters above the brackets represent statistically significant differences $(P<0.05)$. induction after inoculation by B. cinerea (Li et al. 2012; Ren et al. 2008). There are several possible explanations for these seemingly paradoxical findings. First, MPK3/MPK6-regulated GLIP1, GLIP3, and GLIP4 expression will be influenced by two pathways, the MPK3/MPK6-mediated phosphorylation of key transcriptional activators such as WRKY33 and dephosphorylation of these transcription factors by one or more unknown phosphatases. Both MPK3 and MPK6 are required to conquer the activity of their phosphatases and effectively keep the phosphorylation of downstream transcription factors to subsequently drive GLIP1, GLIP3, and GLIP4 expression in $D D$ plants. When either $M P K 3$ or $M P K 6$ is mutant, the phosphorylation strength is unable to counteract the dephosphorylation action and GLIP1, GLIP3, and GLIP4 expression is severely inhibited. In contrast, $B$. cinerea infection not only activates the MPK3/MPK6 pathway (Li et al. 2012), leading to WRKY33 phosphorylation, but may also inactivate its dephosphorylation process. As a result, the absence of either MAPK may not be able to suppress GLIP1, GLIP3, and GLIP4 expression. An alternative explanation is that $B$. cinerea induction can activate various signaling pathways in plants. In addition to the MAPK cascade, the activation of other pathways can probably make up for the weakened MAPK cascade in both $m p k 3$ and $m p k 6$ single mutants, which makes it essential to employ a mpk3 mpk6 double-mutant system to observe the loss-of-function phenotype after $B$. cinerea sporeinoculation.

\section{WRKY transcription factors function downstream of MPK3 and MPK6 in modulation of GLIP1 gene expression.}

Accumulating evidence revealed the crucial function of MPK3 and MPK6 in the regulation of a range of pathogentriggered defense responses (Guan et al. 2015; Lassowskat et al. 2014; Lee et al. 2015; Meng and Zhang 2013; Meng et al. 2013;

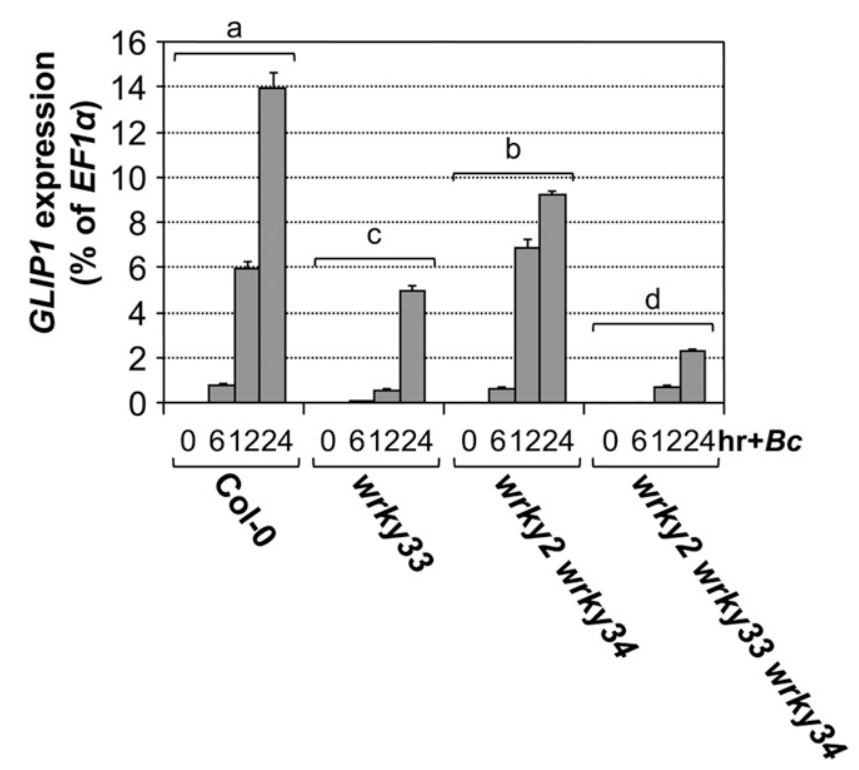

Fig. 6. WRKY2 and WRKY34 play a slight role in the MPK3/MPK6regulated GLIP1 expression in response to Botrytis cinerea. Wild-type (Col-0), wrky33, wrky2 wrky34, and wrky2 wrky33 wrky34 seedlings (12 days old) were treated with $B$. cinerea $(B C)$ spores. Samples were collected at indicated times. Real-time polymerase chain reaction was used to quantify the expression of the GLIP1 gene. GLIP1 transcript level was calculated as a percentage of the EFI $\alpha$ transcript. Error bars represent standard deviations $(n=3)$. Two-way analysis of variance was performed to compare the level of GLIP1 gene expression in different genotypes after $B$. cinerea inoculation. Different lowercase letters above the brackets represent statistically significant differences $(P<0.05)$. 
Rasmussen et al. 2012; Rodriguez et al. 2010; Sheikh et al. 2016; Su et al. 2017; Tsuda et al. 2009, 2013; Xu et al. 2016). The multifunctionality of MPK3 and MPK6 can be attributed to their capacity to phosphorylate distinct substrates. MPK3- and MPK6-mediated phosphorylation of several ACS isoforms can stabilize these ACS proteins and induce ethylene production (Han et al. 2010; Joo et al. 2008; Liu and Zhang 2004; Xu and Zhang 2015b). WRKY33 phosphorylated by MPK3/MPK6 upregulates the camalexin synthetic gene expression and drives the induction of camalexin production by responding to invading pathogen. WRKY33 is also implicated in the transcriptional modulation of ACS 2 and ACS6, two ACS isoforms, which results in abundant ethylene generation by fungi (Li et al. 2012; Mao et al. 2011). Besides WRKYs, MPK3 and MPK6 also phosphorylate other transcription factors, for instance, ERF6 (Meng et al. 2013). Phosphorylated ERF6 activates the transcription of defense genes such as PDF1.1 and PDF1.2a and contributes to plant resistance to fungal pathogen (Meng et al. 2013). Recently, we found the MPK3/MPK6-ERF6 pathway plays critical roles in the dynamic metabolism of IGSs by regulating several key regulators, such as $M Y B 51$ and $M Y B 122$, and key biosynthesis genes, including $C Y P 81 F 2$, $I G M T 1$, and IGMT2 (Xu et al. 2016).

Defense-related GLIP1 expression is dependent on WRKY33 downstream of MPK3 and MPK6 (Fig. 5). WRKY33, as a transcription factor, specifically binds to the C/TTGACT/C DNA motif (W-box) in the promoters of its target genes (Ciolkowski et al. 2008; Pandey and Somssich 2009). ChIP-qPCR results indicated the direct binding of WRKY33 to GLIP1 promoter, which contributes to MPK3- and MPK6-dependent GLIP1 expression after $B$. cinerea inoculation (Fig. 7). However, a recent ChIP-Seq analysis of flg22-treated plants failed to identify GLIP1 as a target of WKY33 (Birkenbihl et al. 2017). This could be due to the sensitivity difference between ChIPqPCR and ChIP-Seq. We found that the flg22-induced GLIPI expression was very low in comparison with the pathogen incubation (Supplementary Fig. S8), which possibly is a result of transient activation of MPK3 and MPK6 by responding to flg22. Both $B$. cinerea and flg22 can trigger activation of MPK3 and MPK6, but the activation kinetics are different between the two treatments. Plant sensing of $B$. cinerea infection triggers a long-lasting MPK3 and MPK6 activation, which leads to high-level camalexin accumulation (Mao et al. 2011; Ren et al. 2008). Conversely, plants treated with flg22 only show a transient activation of MPK3 and MPK6 (Liu and Zhang 2004; Xu et al. 2014), which is not sufficient in inducing camalexin biosynthesis (Millet et al. 2010; Schenke et al. 2011).

\section{Plant defense-related hormones in the activation of GLIP1 gene expression.}

Plant hormones, including salicylic acid (SA), ethylene, and jasmonic acid (JA), play important roles in mediating plant defense response against pathogens (Pieterse et al. 2012). In general, SA signaling is implicated in plant defense against the biotrophic pathogens, while JA and ethylene are involved in the necrotrophic pathogens (Glazebrook 2005). There is a competitive intercommunication or crosstalk between the signaling pathways of SA and JA, such as the SA antagonistic effects on JA-responsive genes (Caarls et al. 2015, 2017; Shigenaga et al. 2017; Verma et al. 2016). On the other hand, JA and ethylene function synergistically in plant immunity (Broekgaarden et al. 2015).

Arabidopsis MPK3 and MPK6 are involved in both the modulation of ethylene synthesis and JA signaling in plant immunity (Guan et al. 2015; Han et al. 2010; Li et al. 2012; Liu and Zhang 2004; Takahashi et al. 2007). Plants challenged by $B$. cinerea generate high levels of ethylene, which is dependent on the MKP3/MPK6 cascade (Han et al. 2010; Li et al. 2012; Liu and Zhang 2004). Here, we found MPK3- and MPK6-mediated GLIP1 expression is not dependent on ethylene signaling when plants are infected with $B$. cinerea (Fig. 8B). This seems to conflict with results reported in previous publications (Kim et al. 2013; Kwon et al. 2009; Oh et al. 2005), but the opposite conclusion we reached may suggest that the role of ethylene signaling components in induction of GLIPI is subtle and influenced by experimental conditions. Otherwise, the ethylene signaling may play differential roles in regulating $G L I P 1$ expression in response to different biotic stimulus. MPK3- and MPK6-mediated GLIP1 expression is also independent in JA signaling against the pathogen B. cinerea (Fig. 8D), which is consistent with previous results (Kwon et al. 2009; Oh et al. 2005). Previous reports showed that the participation of GLIP1 in Arabidopsis SAR as a SAresponsive secreted protein (Kim et al. 2014; Kwon et al. 2009; Oh et al. 2005). Whether MPK3- and MPK6-mediated GLIP1 expression involves SA signaling remains to be further investigated.

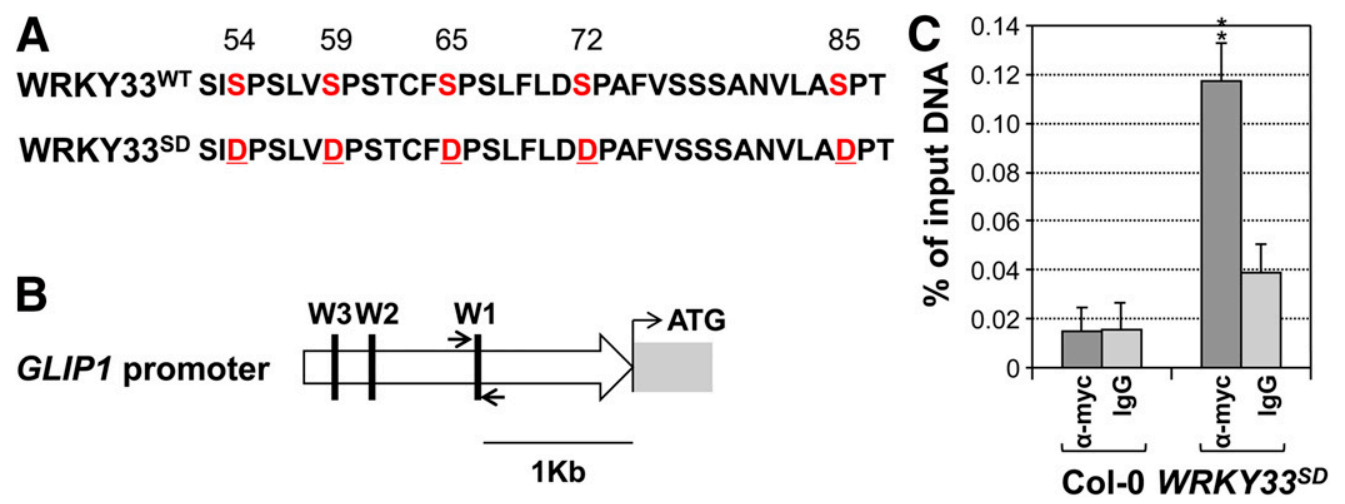

Fig. 7. WRKY33 transcription factor binds to the promoter of the GLIP1 gene in vivo. A, Mitogen-activated protein kinase phosphorylation sites in the N terminus of WRKY33 and the phospho-mimicking WRKY33 protein with all five serine (S) mutated to aspartic acid (D) (WRKY33 ${ }^{\text {SD }}$ ). B, The number and relative position of the W-boxes in the GLIP1 promoter. Line arrows indicate the position of primers used for quantitative polymerase chain reaction ( $\mathrm{qPCR}$ ) after chromatin immunoprecipitation (ChIP). C, WRKY33 binds to the promoter of the GLIP1 gene in vivo. ChIP-qPCR analysis was performed using 12-dayold Col-0 and 35S:WRKY33 $/$ wrky33 seedlings inoculated with B. cinerea spores for 12 h. Tagged $4 \mathrm{myc}-\mathrm{WRKY} 33^{\mathrm{SD}}$ protein-chromatin complex was immunoprecipitated with an antimyc antibody. A control reaction was processed side-by-side, using mouse immunoglobulin G (IgG). ChIP- and input-DNA samples were quantified by real-time $\mathrm{qPCR}$, using primers specific to the W-box-containing region (B). The ChIP results are presented as a percentage of input DNA. Error bars indicate standard deviations $(n=3)$. Asterisks represent significant differences in DNA abundance of antimyc and IgG antibody in the same seedlings (Student's $t$ test, asterisks [**] indicate $P<0.01$ ). 


\section{MATERIALS AND METHODS}

Plant materials.

Columbia (Col-0) ecotype is applied as the background of all Arabidopsis thaliana mutants and transgenic lines in this work. Steroid-inducible promoter-driven tobacco $G V G-N t M E K 2^{D D}$ transgenic Arabidopsis plants (DD) (Ren et al. 2002), glip1-1 and glipl-2 (Oh et al. 2005), mpk3-1 (Wang et al. 2007), mpk62 (Liu and Zhang 2004), wrky33-1 and wrky33-2 (Zheng et al. 2006), etr1-1 (Chang et al. 1993), ein2-1 (Roman et al. 1995), ein3-1 (Chao et al. 1997), ctr1-1 (Kieber et al. 1993), and coil1 (Xie et al. 1998) mutants were described previously. $D D$ mpk3 (Wang et al. 2007), DD mpk6 (Liu and Zhang 2004), 35S: $4 m y c-W R K Y 33^{W T} /$ wrky33 (Mao et al. 2011), wrky2 wrky34 (Guan et al. 2014), as well as chemical genetically rescued mpk3 mpk6 double mutants (MPK6SR and MPK3SR) (Xu et al. 2014, 2016) were previously described . The T-DNA insertion alleles glip3-1 (SALK_201623C), glip4-1 (WiscDsLox489492B15), and glip4-2 (SALK_048818) were obtained from the Arabidopsis Biological Resource Center.

For mutant genotyping, the LBb1.3 (5'-ATTTTGCCGAT TTCGGAAC- $\left.3^{\prime}\right)$ and P745 (5'-AACGTCCGCAATGTGTTAT TAAGTTGTC-3') border primers were used to detect the SALK lines (glip3-1 and glip4-2) and the Wisconsin line (glip4-1), respectively. The gene-specific primers used were as follows: GLIP3-LP, 5'-AGAACAACAAACTTGACCATGC$3^{\prime}$ and GLIP3-RP, 5'-CATGAAGGGCGTATCTAAAACC-3' for the glip3-1 mutant; GLIP4-LP, 5'-TGATTATTGA CGCTGTCTTCGT-3' and GLIP4-RP, 5'-ACCGTAACGAG AAGGATTGTTG-3' for glip4-1 and glip4-2 mutants.

\section{Plant growth conditions and treatment.}

Arabidopsis seedlings were grown and treated as described before (Mao et al. 2011). Arabidopsis seeds were maintained, surface-sterilized, and vernalized for about 3 to 5 days at $4^{\circ} \mathrm{C}$. All the seeds were sown in petri dishes containing liquid halfstrength Murashige and Skoog (MS) media and were incubated under continuous light at $22^{\circ} \mathrm{C}$ in a growth chamber as described before (Li et al. 2012). Seedlings were transferred 5 days later to 20 -ml gas chromatography (GC) vials containing $6 \mathrm{ml}$ of the same liquid media (10 seedlings for each vial) and were retained under similar growth circumstances. The 12-dayold seedlings that were grown in GC vials were used for treatment. The seedlings were collected at various times after utilization of DEX (final concentration of $1 \mu \mathrm{M}$ ), inoculation of Botrytis cinerea spores $\left(4.0 \times 10^{5}\right.$ spores in each vial), ACC (1 or $10 \mu \mathrm{M})$, or MeJA (50 or $100 \mu \mathrm{M})$. Processes for maintenance of $B$. cinerea as well as preparation of spores were as earlier indicated (Han et al. 2010; Li et al. 2012; Ren et al. 2008).

\section{Generation of transgenic plants.}

To generate the phospho-mimicking WRKY33 variant (WRKY33 ${ }^{\mathrm{SD}}$ ), the wild-type WRKY33 complementary (c) DNA was amplified via PCR, using primers WRKY33-F1, 5' ATGGCTGCTTCTTTTCTTACAATGG-3' and WRKY33-B1, 5'-TCAGGGCATAAACGAATCGAAAAATGAGG-3' and was transferred into the vector pBluescript II KS. Site-directed mutagenesis was performed to get the mutations in the phosphorylation sites of WRKY33. The mutations were verified via sequencing. Briefly, the five target phosphorylation sites of WRKY33, serine54, serine-59, serine-65, serine-72, and serine-85, were designated as $1,2,3,4$, and 5, respectively. Primers that were utilized for mutagenesis were presented as below: 12DDF1, 5'-CTC CTTCTTCAATCTCTATCGATCCTTCTCTTGTCGATCCTT CCACTTGTTTC-3'; 34DDF1, $5^{\prime}$-ATCCTTCCACTTGTTTCGA TCCCTCTCTTTTTCTCGATGACCCTGCTTTTGTCTCC-3'; 5DF1, 5'-CTCTGCTAACGTTCTAGCTGATCCAACCACAGG
AGC-3', as well as their complementary primers. The mutated nucleotides are in bold and underlined. We generated the WRKY $33^{\text {SD }}$ with all five serine residues mutated to aspartic acid residues, according to three sequential mutagenesis steps.

For the purposes of establishing the $35 S: 4 m y c-W R K Y 33^{S D}$ construct, we amplified the WRKY $33^{\text {SD }}$ cDNA fragment using the WRKY33-F1/WRKY33-B1 primer pair and subcloned this fragment into an altered vector pBluescript II KS with the Nterminal 4 myc tag. This 4 myc-tagged WRKY $33^{\mathrm{SD}}$ cDNA was shifted into modified vector pBI121 by using the SpeI/XhoI sites. In addition, transformation of Agrobacterium tumefaciens GV3101 that carried the construct was implemented into Arabidopsis using a floral-dipping approach (Clough and Bent 1998). Immunoblot analyses were used to select independent lines expressing 4myc-tagged WRKY33 ${ }^{\text {SD }}$. Successfully transformed plants comprising a single copy of T-DNA insertion were identified on the basis of $3: 1$ segregation of kanamycin resistance in the T2 generation. Homozygous transgenic lines in the T3 generation were applied for further experiments.
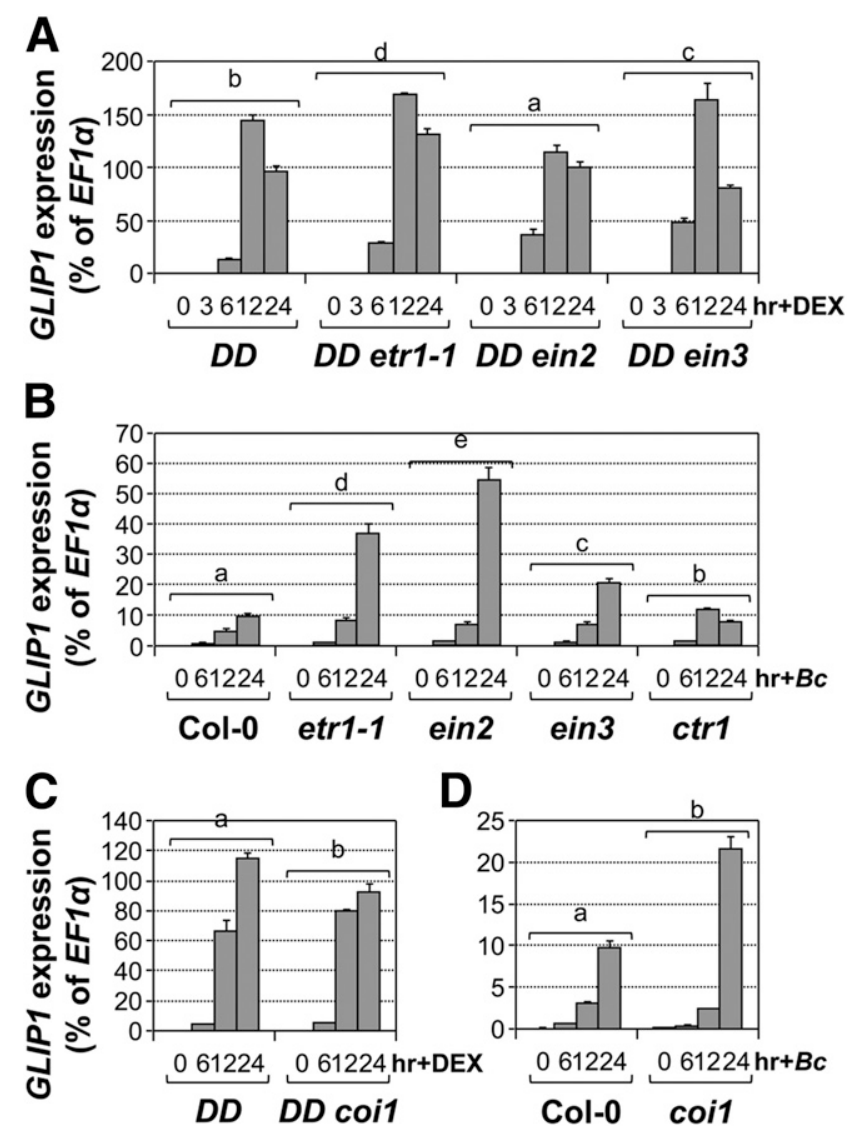

Fig. 8. MPK3/MPK6-induced GLIP1 expression is independent of ethylene and jasmonic acid. A, The 12-day-old GVG-NtMEK2 $2^{D D}(D D)$, DD etr1-1, $D D$ ein 2 , and $D D$ ein 3 seedlings were treated with $1 \mu \mathrm{M}$ dexamethasone (DEX), and B, 12-day-old wild-type (Col-0), etr1-1, ein2, ein3, and ctr 1-1 seedlings were inoculated with Botrytis cinerea $(B c)$ spores. $\mathbf{C}, D D$ and $D D$ coil seedlings (12 days old) were treated with $1 \mu \mathrm{M}$ DEX, and D, 12-dayold wild-type (Col-0) and coil seedlings were inoculated with $B$. cinerea spores. Samples were collected at indicated times. Real-time polymerase chain reaction was used to quantify the expression of the GLIP1 gene. The GLIP1 transcript level was calculated as a percentage of the EF1 $\alpha$ transcript. Error bars represent standard deviations $(n=3)$. Two-way analysis of variance was performed to compare the level of GLIP1 gene expression in different genotypes after DEX or B. cinerea treatment. Different lowercase letters above the brackets represent statistically significant differences $(P<0.05)$. 


\section{RNA extraction and real-time PCR analysis.}

The extraction of total plant RNA was performed using the TRIzol reagent (Invitrogen). After treatment with DNase (Invitrogen), total RNA ( $1 \mu \mathrm{g})$ was deployed to synthesize firststrand cDNA. RT-PCR and real-time PCR were performed to characterize the T-DNA insertion mutants and gene expression, respectively. A real-time PCR machine (Eppendorf) was used to conduct a real-time PCR analysis as presented by Ren et al. (2008). Normalization of samples was performed using the reference gene $E F 1 \alpha$. The expression levels of genes were evaluated utilizing two different approaches. The first approach was the commonly used $2^{-\Delta \Delta \mathrm{Ct}}$ cycle threshold method offering fold of gene induction that is relative to basal level before treatment $(0 \mathrm{~h}$ timepoint $)$. The other approach shows the transcript level that is relative to the EFl $\alpha$ gene in the same sample, which is deemed a superior method to compare the expression levels among diverse genes. The primers that were applied in real-time PCR and RT-PCR are presented in Supplementary Table S1.

\section{B. cinerea resistance assay.}

For the $B$. cinerea resistance assay, seeds were sown in soil and were maintained in a growth chamber at $22^{\circ} \mathrm{C}$ and about $65 \%$ relative humidity under a 10 -h light and 14-h dark cycle for 4 weeks. Detailed procedures of $B$. cinerea maintenance and spore preparation were as described (Ferrari et al. 2003; Meng et al. 2013). Mature rosette leaves detached from 4-week-old plants were placed in petri dishes containing $0.8 \%$ agar, with the petiole embedded in the medium. Each leaf was inoculated with a $10-\mu l$ drop of spore suspension $\left(5 \times 10^{5}\right.$ spores $\left./ \mathrm{ml}\right)$. Leaves were photographed and lesion size was measured at 3 days after inoculation.

\section{ChIP-qPCR analysis.}

ChIP-qPCR processing and assay was implemented as presented before (Xu et al. 2016). The 12-day-old seedlings inoculated with $B$. cinerea spores for $12 \mathrm{~h}$ were harvested for this experiment. First, these samples were subjected to crosslinking and processing as described by Kaufmann et al. (2010). One gram of the resulting tissue was used to isolate the chromatin, and the chromatin was then sheared with an enzymatic shearing kit (Active Motif). Subsequently, immunoprecipitation was performed by incubation of chromatin samples with $2 \mu \mathrm{g}$ of antimyc antibody (Millipore) or mouse IgG (negative control) at $4^{\circ} \mathrm{C}$ for about $1 \mathrm{~h}$. The protein-chromatin immunocomplexes were captured by ultilization of Protein G-Dynal magnetic beads (Invitrogen). Purification of the immunoprecipitated DNA was performed after Proteinase $\mathrm{K}$ digestion with a ChIP DNA clean and concentrator kit (Zymo Research). The immunoprecipitated DNA and imput DNA were then subjected to $\mathrm{qPCR}$ with the primers that were specific for the promoter regoin of GLIP1. These forward and backward primer pairs that were ultilized for the purpose of ChIP-qPCR included 5' CGAGTGAACAAACGAAGCA-3' and 5' -TCGGAAAATGCC GTCTAGT-3', both of which are near the W-boxes in the GLIPI promoter. The ChIP-qPCR outcomes are expressed as the percentage of input DNA.

\section{Statistical analyses.}

All the experiments mentioned in this research were performed independently at least three times with similar outcomes, and one representative dataset is shown. GraphPad Prism 6.0 was used to perform statistical analyses. The differences are statistically significant $(P<0.05)$, very significant $(P<0.01)$, or extremely significant $(P<0.001)$ as indicated by single, double, or triple asterisks, respectively (Student's $t$ test). Tukey's post hoc test was used to perform a two-way analysis of variance when time-course data of different mutants were compared (Brady et al. 2015). Statistically significant differences $(P<0.05)$ are shown in figures by different letters above the brackets.

\section{LITERATURE CITED}

Akoh, C. C., Lee, G.-C., Liaw, Y.-C., Huang, T.-H., and Shaw, J.-F. 2004 GDSL family of serine esterases/lipases. Prog. Lipid Res. 43:534-552.

Asai, T., Tena, G., Plotnikova, J., Willmann, M. R., Chiu, W.-L., GomezGomez, L., Boller, T., Ausubel, F. M., and Sheen, J. 2002. MAP kinase signalling cascade in Arabidopsis innate immunity. Nature 415:977-983.

Bacete, L., Mélida, H., Miedes, E., and Molina, A. 2018. Plant cell wallmediated immunity: Cell wall changes trigger disease resistance responses. Plant J. 93:614-636.

Bethke, G., Pecher, P., Eschen-Lippold, L., Tsuda, K., Katagiri, F., Glazebrook, J., Scheel, D., and Lee, J. 2012. Activation of the Arabidopsis thaliana mitogen-activated protein kinase MPK11 by the flagellin-derived elicitor peptide, flg22. Mol. Plant-Microbe Interact. 25: 471-480.

Bigeard, J., and Hirt, H. 2018. Nuclear signaling of plant MAPKs. Front. Plant Sci. 9:469.

Birkenbihl, R. P., Kracher, B., and Somssich, I. E. 2017. Induced genomewide binding of three Arabidopsis WRKY transcription factors during early MAMP-triggered immunity. Plant Cell 29:20-38.

Bishop, A. C., Ubersax, J. A., Petsch, D. T., Matheos, D. P., Gray, N. S., Blethrow, J., Shimizu, E., Tsien, J. Z., Schultz, P. G., Rose, M. D., Wood, J. L., Morgan, D. O., and Shokat, K. M. 2000. A chemical switch for inhibitor-sensitive alleles of any protein kinase. Nature 407:395-401.

Boller, T., and Felix, G. 2009. A renaissance of elicitors: Perception of microbe-associated molecular patterns and danger signals by patternrecognition receptors. Annu. Rev. Plant Biol. 60:379-406.

Brady, S. M., Burow, M., Busch, W., Carlborg, Ö., Denby, K. J., Glazebrook, J., Hamilton, E. S., Harmer, S. L., Haswell, E. S., Maloof, J. N., Springer, N. M., and Kliebenstein, D. J. 2015. Reassess the $t$ test: Interact with all your data via ANOVA. Plant Cell 27:2088-2094.

Brick, D. J., Brumlik, M. J., Buckley, J. T., Cao, J.-X., Davies, P. C., Misra, S., Tranbarger, T. J., and Upton, C. 1995. A new family of lipolytic plant enzymes with members in rice, Arabidopsis and maize. FEBS Lett. 377: 475-480.

Broekgaarden, C., Caarls, L., Vos, I. A., Pieterse, C. M. J., and Van Wees, S. C. M. 2015. Ethylene: Traffic controller on hormonal crossroads to defense. Plant Physiol. 169:2371-2379.

Caarls, L., Pieterse, C. M., and Van Wees, S. C. 2015. How salicylic acid takes transcriptional control over jasmonic acid signaling. Front. Plant Sci. 6:170.

Caarls, L., Van der Does, D., Hickman, R., Jansen, W., Verk, M. C., Proietti, S., Lorenzo, O., Solano, R., Pieterse, C. M., and Van Wees, S. C. 2017. Assessing the role of ETHYLENE RESPONSE FACTOR transcriptional repressors in salicylic acid-mediated suppression of jasmonic acidresponsive genes. Plant Cell Physiol. 58:266-278.

Chang, C., Kwok, S. F., Bleecker, A. B., and Meyerowitz, E. M. 1993. Arabidopsis ethylene-response gene ETR1: Similarity of product to twocomponent regulators. Science 262:539-544.

Chao, Q., Rothenberg, M., Solano, R., Roman, G., Terzaghi, W., and Ecker, J. R. 1997. Activation of the ethylene gas response pathway in Arabidopsis by the nuclear protein ETHYLENE-INSENSITIVE3 and related proteins. Cell 89:1133-1144.

Chen, Z., Gibson, T. B., Robinson, F., Silvestro, L., Pearson, G., Xu, B., Wright, A., Vanderbilt, C., and Cobb, M. H. 2001. MAP kinases. Chem. Rev. 101:2449-2476.

Ciolkowski, I., Wanke, D., Birkenbihl, R. P., and Somssich, I. E. 2008 Studies on DNA-binding selectivity of WRKY transcription factors lend structural clues into WRKY-domain function. Plant Mol. Biol. 68:81-92.

Clough, S. J., and Bent, A. F. 1998. Floral dip: A simplified method for Agrobacterium-mediated transformation of Arabidopsis thaliana. Plant J. 16:735-743.

Colcombet, J., and Hirt, H. 2008. Arabidopsis MAPKs: A complex signalling network involved in multiple biological processes. Biochem. J. 413:217-226.

Coll, N. S., Epple, P., and Dangl, J. L. 2011. Programmed cell death in the plant immune system. Cell Death Differ. 18:1247-1256.

Dixon, R. A. 2001. Natural products and plant disease resistance. Nature 411:843-847.

Dóczi, R., Okrész, L., Romero, A. E., Paccanaro, A., and Bögre, L. 2012. Exploring the evolutionary path of plant MAPK networks. Trends Plant Sci. 17:518-525. 
Ferrari, S., Plotnikova, J. M., De Lorenzo, G., and Ausubel, F. M. 2003. Arabidopsis local resistance to Botrytis cinerea involves salicylic acid and camalexin and requires EDS4 and PAD2, but not SID2, EDS5 or PAD4. Plant J. 35:193-205.

Gao, M., Liu, J., Bi, D., Zhang, Z., Cheng, F., Chen, S., and Zhang, Y. 2008 MEKK1, MKK1/MKK2 and MPK4 function together in a mitogenactivated protein kinase cascade to regulate innate immunity in plants. Cell Res. 18:1190-1198.

Gao, M., Yin, X., Yang, W., Lam, S. M., Tong, X., Liu, J., Wang, X., Li, Q., Shui, G., and He, Z. 2017. GDSL lipases modulate immunity through lipid homeostasis in rice. PLoS Pathog. 13:e1006724.

Gfeller, A., Liechti, R., and Farmer, E. E. 2010. Arabidopsis jasmonate signaling pathway. Sci. Signal. 3:cm4

Glazebrook, J. 2005. Contrasting mechanisms of defense against biotrophic and necrotrophic pathogens. Annu. Rev. Phytopathol. 43:205-227.

Guan, R., Su, J., Meng, X., Li, S., Liu, Y., Xu, J., and Zhang, S. 2015. Multilayered regulation of ethylene induction plays a positive role in Arabidopsis resistance against Pseudomonas syringae. Plant Physiol. 169:299-312.

Guan, Y., Meng, X., Khanna, R., LaMontagne, E., Liu, Y., and Zhang, S. 2014. Phosphorylation of a WRKY transcription factor by MAPKs is required for pollen development and function in Arabidopsis. PLoS Genet. 10:e1004384

Han, L., Li, G.-J., Yang, K.-Y., Mao, G., Wang, R., Liu, Y., and Zhang, S 2010. Mitogen-activated protein kinase 3 and 6 regulate Botrytis cinerea-induced ethylene production in Arabidopsis. Plant J. 64:114-127.

Ichimura, K., Casais, C., Peck, S. C., Shinozaki, K., and Shirasu, K. 2006. MEKK1 is required for MPK4 activation and regulates tissue-specific and temperature-dependent cell death in Arabidopsis. J. Biol. Chem. 281:36969-36976.

Joo, S., Liu, Y., Lueth, A., and Zhang, S. 2008. MAPK phosphorylationinduced stabilization of ACS6 protein is mediated by the non-catalytic C-terminal domain, which also contains the cis-determinant for rapid degradation by the 26S proteasome pathway. Plant J. 54:129-140.

Jwa, N.-S., and Hwang, B. K. 2017. Convergent evolution of pathogen effectors toward reactive oxygen species signaling networks in plants. Front. Plant Sci. 8:1687.

Kaufmann, K., Muiño, J. M., Østerås, M., Farinelli, L., Krajewski, P., and Angenent, G. C. 2010. Chromatin immunoprecipitation (ChIP) of plant transcription factors followed by sequencing (ChIP-SEQ) or hybridization to whole genome arrays (ChIP-CHIP). Nat. Protoc. 5:457-472.

Kieber, J. J., Rothenberg, M., Roman, G., Feldmann, K. A., and Ecker, J. R. 1993. CTR1, a negative regulator of the ethylene response pathway in Arabidopsis, encodes a member of the raf family of protein kinases. Cell 72:427-441.

Kim, C. Y., Liu, Y., Thorne, E. T., Yang, H., Fukushige, H., Gassmann, W. Hildebrand, D., Sharp, R. E., and Zhang, S. 2003. Activation of a stressresponsive mitogen-activated protein kinase cascade induces the biosynthesis of ethylene in plants. Plant Cell 15:2707-2718.

Kim, H. G., Kwon, S. J., Jang, Y. J., Chung, J. H., Nam, M. H., and Park O. K. 2014. GDSL lipase 1 regulates ethylene signaling and ethyleneassociated systemic immunity in Arabidopsis. FEBS Lett. 588: 1652-1658.

Kim, H. G., Kwon, S. J., Jang, Y. J., Nam, M. H., Chung, J. H., Na, Y. C., Guo, H., and Park, O. K. 2013. GDSL LIPASE1 modulates plant immunity through feedback regulation of ethylene signaling. Plant Physiol. 163:1776-1791.

Kong, Q., Qu, N., Gao, M., Zhang, Z., Ding, X., Yang, F., Li, Y., Dong, O. X., Chen, S., Li, X., and Zhang, Y. 2012. The MEKK1MKK1/MKK2-MPK4 kinase cascade negatively regulates immunity mediated by a mitogen-activated protein kinase kinase kinase in Arabidopsis. Plant Cell 24:2225-2236.

Kotchoni, S. O., and Gachomo, E. W. 2006. The reactive oxygen species network pathways:an essential prerequisite for perception of pathogen attack and the acquired disease resistance in plants. J. Biosci. 31: 389-404.

Kwon, S. J., Jin, H. C., Lee, S., Nam, M. H., Chung, J. H., Kwon, S. I., Ryu, C. M., and Park, O. K. 2009. GDSL lipase-like 1 regulates systemic resistance associated with ethylene signaling in Arabidopsis. Plant J. 58: $235-245$.

Lassowskat, I., Böttcher, C., Eschen-Lippold, L., Scheel, D., and Lee, J. 2014. Sustained mitogen-activated protein kinase activation reprograms defense metabolism and phosphoprotein profile in Arabidopsis thaliana. Front. Plant Sci. 5:554.

Lee, D. S., Kim, B. K., Kwon, S. J., Jin, H. C., and Park, O. K. 2009 Arabidopsis GDSL lipase 2 plays a role in pathogen defense via negative regulation of auxin signaling. Biochem. Biophys. Res. Commun. 379: 1038-1042.
Lee, J., Eschen-Lippold, L., Lassowskat, I., Böttcher, C., and Scheel, D. 2015. Cellular reprogramming through mitogen-activated protein kinases. Front. Plant Sci. 6:940.

Li, G., Meng, X., Wang, R., Mao, G., Han, L., Liu, Y., and Zhang, S. 2012. Dual-level regulation of ACC synthase activity by MPK3/MPK6 cascade and its downstream WRKY transcription factor during ethylene induction in Arabidopsis. PLoS Genet. 8:e1002767.

Liu, Y., and Zhang, S. 2004. Phosphorylation of 1-aminocyclopropane-1carboxylic acid synthase by MPK6, a stress-responsive mitogenactivated protein kinase, induces ethylene biosynthesis in Arabidopsis. Plant Cell 16:3386-3399.

Mao, G., Meng, X., Liu, Y., Zheng, Z., Chen, Z., and Zhang, S. 2011. Phosphorylation of a WRKY transcription factor by two pathogenresponsive MAPKs drives phytoalexin biosynthesis in Arabidopsis. Plan Cell 23:1639-1653.

Meng, X., Xu, J., He, Y., Yang, K.-Y., Mordorski, B., Liu, Y., and Zhang, S. 2013. Phosphorylation of an ERF transcription factor by Arabidopsis MPK3/MPK6 regulates plant defense gene induction and fungal resistance. Plant Cell 25:1126-1142.

Meng, X., and Zhang, S. 2013. MAPK cascades in plant disease resistance signaling. Annu. Rev. Phytopathol. 51:245-266.

Millet, Y. A., Danna, C. H., Clay, N. K., Songnuan, W., Simon, M. D., Werck-Reichhart, D., and Ausubel, F. M. 2010. Innate immune responses activated in Arabidopsis roots by microbe-associated molecular patterns. Plant Cell 22:973-990.

Oh, I. S., Park, A. R., Bae, M. S., Kwon, S. J., Kim, Y. S., Lee, J. E., Kang, N. Y., Lee, S., Cheong, H., and Park, O. K. 2005. Secretome analysis reveals an Arabidopsis lipase involved in defense against Alternaria brassicicola. Plant Cell 17:2832-2847.

Pandey, S. P., and Somssich, I. E. 2009. The role of WRKY transcription factors in plant immunity. Plant Physiol. 150:1648-1655.

Pedley, K. F., and Martin, G. B. 2005. Role of mitogen-activated protein kinases in plant immunity. Curr. Opin. Plant Biol. 8:541-547.

Pieterse, C. M., Van der Does, D., Zamioudis, C., Leon-Reyes, A., and Van Wees, S. C. 2012. Hormonal modulation of plant immunity. Annu. Rev. Cell Dev. Biol. 28:489-521.

Pitzschke, A. 2015. Modes of MAPK substrate recognition and control. Trends Plant Sci. 20:49-55.

Qiu, J.-L., Zhou, L., Yun, B.-W., Nielsen, H. B., Fiil, B. K., Petersen, K., Mackinlay, J., Loake, G. J., Mundy, J., and Morris, P. C. 2008 Arabidopsis mitogen-activated protein kinase kinases MKK1 and MKK2 have overlapping functions in defense signaling mediated by MEKK1, MPK4, and MKS1. Plant Physiol. 148:212-222.

Rasmussen, M. W., Roux, M., Petersen, M., and Mundy, J. 2012. MAP kinase cascades in Arabidopsis innate immunity. Front. Plant Sci. 3:169.

Ren, D., Liu, Y., Yang, K.-Y., Han, L., Mao, G., Glazebrook, J., and Zhang, S. 2008. A fungal-responsive MAPK cascade regulates phytoalexin biosynthesis in Arabidopsis. Proc. Natl. Acad. Sci. U.S.A. 105:5638-5643.

Ren, D., Yang, H., and Zhang, S. 2002. Cell death mediated by MAPK is associated with hydrogen peroxide production in Arabidopsis. J. Biol. Chem. 277:559-565.

Rodriguez, M. C., Petersen, M., and Mundy, J. 2010. Mitogen-activated protein kinase signaling in plants. Annu. Rev. Plant Biol. 61:621-649.

Roman, G., Lubarsky, B., Kieber, J. J., Rothenberg, M., and Ecker, J. R. 1995. Genetic analysis of ethylene signal transduction in Arabidopsis thaliana: Five novel mutant loci integrated into a stress response pathway. Genetics 139:1393-1409.

Schenke, D., Böttcher, C., and Scheel, D. 2011. Crosstalk between abiotic ultraviolet-B stress and biotic (flg22) stress signalling in Arabidopsis prevents flavonol accumulation in favor of pathogen defence compound production. Plant Cell Environ. 34:1849-1864.

Sheikh, A. H., Eschen-Lippold, L., Pecher, P., Hoehenwarter, W., Sinha, A. K., Scheel, D., and Lee, J. 2016. Regulation of WRKY46 transcription factor function by mitogen-activated protein kinases in Arabidopsis thaliana. Front. Plant Sci. 7:61.

Shigenaga, A. M., Berens, M. L., Tsuda, K., and Argueso, C. T. 2017. Towards engineering of hormonal crosstalk in plant immunity. Curr Opin. Plant Biol. 38:164-172.

Sticher, L., Mauch-Mani, B., and Métraux, J.-P. 1997. Systemic acquired resistance. Annu. Rev. Phytopathol. 35:235-270.

Su, J., Yang, L., Zhu, Q., Wu, H., He, Y., Liu, Y., Xu, J., Jiang, D., and Zhang, S. 2018. Active photosynthetic inhibition mediated by MPK3/MPK6 is critical to effector-triggered immunity. PLoS Biol. 16: e2004122.

Su, J., Zhang, M., Zhang, L., Sun, T., Liu, Y., Lukowitz, W., Xu, J., and Zhang, S. 2017. Regulation of stomatal immunity by interdependent functions of a pathogen-responsive MPK3/MPK6 cascade and abscisic acid. Plant Cell 29:526-542. 
Suarez-Rodriguez, M. C., Adams-Phillips, L., Liu, Y., Wang, H., Su, S. H., Jester, P. J., Zhang, S., Bent, A. F., and Krysan, P. J. 2007. MEKK1 is required for flg22-induced MPK4 activation in Arabidopsis plants. Plant Physiol. 143:661-669.

Taj, G., Agarwal, P., Grant, M., and Kumar, A. 2010. MAPK machinery in plants: Recognition and response to different stresses through multiple signal transduction pathways. Plant Signal. Behav. 5:1370-1378.

Takahashi, F., Yoshida, R., Ichimura, K., Mizoguchi, T., Seo, S., Yonezawa, M., Maruyama, K., Yamaguchi-Shinozaki, K., and Shinozaki, K. 2007. The mitogen-activated protein kinase cascade MKK3-MPK6 is an important part of the jasmonate signal transduction pathway in Arabidopsis. Plant Cell 19:805-818.

Tsuda, K., Mine, A., Bethke, G., Igarashi, D., Botanga, C. J., Tsuda, Y., Glazebrook, J., Sato, M., and Katagiri, F. 2013. Dual regulation of gene expression mediated by extended MAPK activation and salicylic acid contributes to robust innate immunity in Arabidopsis thaliana. PLoS Genet. 9:e1004015.

Tsuda, K., Sato, M., Stoddard, T., Glazebrook, J., and Katagiri, F. 2009. Network properties of robust immunity in plants. PLoS Genet. 5:e1000772.

Ueda, M., Aichinger, E., Gong, W., Groot, E., Verstraeten, I., Vu, L. D., De Smet, I., Higashiyama, T., Umeda, M., and Laux, T. 2017. Transcriptional integration of paternal and maternal factors in the Arabidopsis zygote. Genes Dev. 31:617-627.

Verma, V., Ravindran, P., and Kumar, P. P. 2016. Plant hormone-mediated regulation of stress responses. BMC Plant Biol. 16:86.

Wang, H., Ngwenyama, N., Liu, Y., Walker, J. C., and Zhang, S. 2007. Stomatal development and patterning are regulated by environmentally responsive mitogen-activated protein kinases in Arabidopsis. Plant Cell 19:63-73.
Xie, D.-X., Feys, B. F., James, S., Nieto-Rostro, M., and Turner, J. G. 1998. COI1: An Arabidopsis gene required for jasmonate-regulated defense and fertility. Science 280:1091-1094.

Xu, J., Meng, J., Meng, X., Zhao, Y., Liu, J., Sun, T., Liu, Y., Wang, Q., and Zhang, S. 2016. Pathogen-responsive MPK3 and MPK6 reprogram the biosynthesis of indole glucosinolates and their derivatives in Arabidopsis immunity. Plant Cell 28:1144-1162.

Xu, J., Xie, J., Yan, C., Zou, X., Ren, D., and Zhang, S. 2014. A chemical genetic approach demonstrates that MPK3/MPK6 activation and NADPH oxidase-mediated oxidative burst are two independent signaling events in plant immunity. Plant J. 77:222-234.

$\mathrm{Xu}$, J., and Zhang, S. 2015a. Mitogen-activated protein kinase cascades in signaling plant growth and development. Trends Plant Sci. 20:56-64.

$\mathrm{Xu}$, J., and Zhang, S. 2015b. Ethylene biosynthesis and regulation in plants. Pages 1-25 in: Ethylene in Plants. C. K. Wen, ed. Springer, Dordrecht, The Netherlands.

Yang, K.-Y., Liu, Y., and Zhang, S. 2001. Activation of a mitogen-activated protein kinase pathway is involved in disease resistance in tobacco. Proc. Natl. Acad. Sci. U.S.A. 98:741-746.

Zhang, M., Su, J., Zhang, Y., Xu, J., and Zhang, S. 2018. Conveying endogenous and exogenous signals: MAPK cascades in plant growth and defense. Curr. Opin. Plant Biol. 45 (Pt A):1-10.

Zhang, M., Wu, H., Su, J., Wang, H., Zhu, Q., Liu, Y., Xu, J., Lukowitz, W., and Zhang, S. 2017. Maternal control of embryogenesis by MPK6 and its upstream MKK4/MKK5 in Arabidopsis. Plant J. 92: 1005-1019.

Zheng, Z., Qamar, S. A., Chen, Z., and Mengiste, T. 2006. Arabidopsis WRKY33 transcription factor is required for resistance to necrotrophic fungal pathogens. Plant J. 48:592-605. 